DIVISION X / 40 - RADIO ASTRONOMY 


\title{
NEW HORIZONS FOR RADIO ASTRONOMY 1993-1996
}

\section{J. B. Whiteoak President IAU Commission 40}

\author{
PRESIDENT: J.B. Whiteoak (Australia) \\ VICE PRESIDENT: J.M. Moran (USA) \\ ORGANIZING COMMITTEE: K.R. Anantharamaiah (India), E.E. Baart (South Africa), \\ L.B. Baath (Sweden), E.M. Berkhuijsen (Germany), F.R. Colomb (Argentina), \\ R.J. Davis (UK), H.R. Dickel (USA), E. Gerard (France), \\ J. Gomez Gonzalez (Spain), I.V. Gosachinskij (Russia), K.J. Johnston (USA), \\ M. Morimoto (Japan), L. Padrielli (Italy), T. Velusamy (USA), S. Ye (China)
}

\section{Forward}

There have been many advances in radio astronomy during the last three years, and a sample of these are highlighted in the following Report. The coverage of all facets of radio astronomy is certainly not uniform, because much of the text has been provided as voluntary contributions by Commission 40 members, and the finished report will therefore be weighted according to individual interests. Nevertheless, hopefully this Report will show the enormous scope of today's radio astronomy, and reflect some of the excitement of the latest findings.

\section{Radio Telescopes, Instrumentation and Techniques}

\subsection{RADIO TELESCOPES}

\subsubsection{Single Aperture Instruments}

Sub-millimetre Wavelength Telescopes. A new 2-mm/3-mm SIS receiver built at the Onsala Space Observatory (OSO) has been a very useful addition to the Swedish-ESO Submillimetre Telescope (SEST). The 3-mm receiver replaced a less-sensitive Schottky diode system, whereas the new 2-mm system opened up a new spectral window on the southern sky. The tuning flexibility of the SEST operating system coupled with the 1-GHz bandwidth of the backend have been used to advantage in the search for molecularline absorption towards lensed galaxies, and the confirmation of possible detections by searches for other transitions with similar redshifts (e.g. PKS 1830-211).

A Japanese group which includes the Nobeyama Radio Observatory (NRO), University of Tokyo, and the Institute for Molecular Science has begun the construction of a 1.2-m sub-mm telescope on Mt Fuji (at elevation $3700 \mathrm{~m}$ ); it will be used for surveys of CI (at 492 and $809 \mathrm{GHz}$ ) and high-sensitivity searches for new interstellar molecules.

Millimetre Wavelength Telescopes. For the 45-m telescope of the NRO, a prototype 4-beam SIS receiver has been used at $100 \mathrm{GHz}$ for large-scale mapping of molecular clouds. A new optical instrument has been constructed to monitor the geometrical deformation of the reflecting surface; this provides real-time estimates of pointing accuracy and antenna efficiency in windy conditions.

A second 3-mm receiver has been installed on the 30-m antenna at the Pico Veleta Observatory of the Institut de Radio Astronomie Millimetrique (IRAM); four-receiver operation is now possible. The new 19channel bolometer supplied by the Max-Planck-Institut für Radioastronomie (MPIfR) has been integrated into the observing system in a way similar to the heterodyne receivers. The frequency-switching mode can now operate with three receivers simultaneously. An on-the-fly observing mode has been implemented, enabling observers to map relatively large areas of sky.

The James Clerk Maxwell Telescope (JCMT) in Hawaii represents a collaboration between the UK, the Netherlands and Canada. The Canadian JCMT group within the Herzberg Institute of Astrophysics provides staffing support for the telescope, builds advanced receivers, and assesses Canadian observing proposals. The group has just completed a dual-polarization $345-\mathrm{GHz}$ receiver which will operate in the frequency range $330-360 \mathrm{GHz}$ and provide nearly an order-of-magnitude improvement over the current 
system. Remote operation will be possible with the system. The group is collaborating with the University of Alberta in the production of a planar array for the telescope.

The 12-m telescope of the US National Radio Astronomy Observatory (NRAO) at Tucson operates with dual-channel SIS receivers providing a near-continuous frequency coverage, excluding atmospheric absorption bands, from 68 to $300 \mathrm{GHz}$. Remote observing capabilities are provided. Observing modes include position, beam and frequency switching, total-power observing, and on-the-fly mapping for both spectral-line and continuum modes. An eight-beam (2x4 array) receiver is available for observations at 220-260 GHz. A polarimeter is available for spectral-line and continuum observations at frequencies near $100 \mathrm{GHz}$.

The 22-m 'Mopra' antenna of the Australia Telescope (AT), operated by the Australian Commonwealth Scientific and Industrial Research Organization (CSIRO), is equipped with a set of 20-cm/13$\mathrm{cm} / 6-\mathrm{cm} / 3.5-\mathrm{cm}$ AT receiving systems. The antenna was used to support the Project Phoenix of the SETI Institute during the first half of 1995 ; the 13-cm system was modified to cover the frequency range $1.2-3.0 \mathrm{GHz}$ and the $20-\mathrm{cm} / 13-\mathrm{cm}$ feed horn was modified to increase the efficiency at the higher frequencies. The inner $15-\mathrm{m}$ of the reflecting surface has solid panels yielding a reasonable efficiency at $\mathrm{mm}$ wavelengths, and a dual-channel SIS receiving system for frequencies between 85 and $116 \mathrm{GHz}$ was installed for testing on the antenna in September 1994. The system is now being used for routine observing, although tuning cannot yet be carried out remotely.

A new 2.5-mm receiver was successfully installed during 1996 on the $13.7-\mathrm{mm}$ radio telescope operated at Delingha, Qinghai province, by the Purple Mountain Observatory.

The University of Nagoya has installed a 4-m telescope at the Las Campanas Observatory in Chile, in a collaboration with the Carnegie Institution of Washington. Operation begun in 1996 using a superconducting $\mathrm{Nb}$ mixer receiver with SSB receiver temperature of $50 \mathrm{~K}$ at $110 \mathrm{GHz}$.

Following a similar project carried out at Nobeyama, a radio astronomy group at the University of Tokyo has installed a 60-cm telescope at the La Silla Observatory of the European Southern Observatory (ESO) in Chile. The prime objective is to carry out a CO J=2-1 survey of the southern galactic plane with a beamwidth ( 9 arcmin) similar to that used for the previous Columbia Survey of CO $\mathrm{J}=1-0$ emission. An SIS receiver and 2048-channel AOS are used on the telescope.

The Instituto Nacional de Astronomia, Optica, y Electronica (INAOE), Mexico, and the University of Massachusetts at Amherst, USA are developing a joint project for the construction of a $50-\mathrm{m}$ telescope, the Gran Telescopio Milimetrico/Large Millimetre Telescope (GMT/LMT), to begin at a high-altitude Mexican site within two years. The reflecting surface will consist of a composite of 126 segments, each of size $5 \mathrm{~m}$; active control systems will maintain high aperture efficiency and pointing accuracy. The antenna will be enclosed in a radome. Operation will be with array receivers in bands within the nominal wavelength range $1-4 \mathrm{~mm}$.

Centimetre to Metre Wavelength Telescopes. The 1000-ft telescope of Arecibo Observatory has undergone a Gregorian feed upgrade in recent years. The first phase involved the construction of a $50-\mathrm{ft}$ high groundscreen surrounding the main reflecting surface, and this suppresses the effects of ground radiation during observations at higher zenith angles. The second phase consisted of the construction of the Gregorian subreflector system within an 85 - $\mathrm{ft}$ aluminium spaceframe which is mounted under the observing platform. The Gregorian system will enable efficient operation at frequencies ranging from $300 \mathrm{MHz}$ to above $10 \mathrm{GHz}$. It will result in higher sensitivity (11 and $8 \mathrm{~K} \mathrm{Jy}^{-1}$ at L-band and C-band respectively) and lower system temperatures than with the old Arecibo systems. Routine operation is expected to begin in early 1997.

The decimetric radio telescope (NRT) of the Nancay Radio Observatory is undergoing a major renovation (the FORT project). This instrument is a transit telescope consisting of a fixed spherical reflector $300 \mathrm{~m}$ long and $35 \mathrm{~m}$ high and a tiltable flat mirror of dimensions $200 \mathrm{~m} \mathrm{x} 40 \mathrm{~m}$. The existing hoghorn feed system is being replaced by an optimized system using a dual-reflector Gregorian antenna designed in collaboration with the CSIRO Division of Radiophysics. The new system will improve the sensitivity of the telescope by a factor of about 2.5. It is expected to be fully operational by the end of 1998 , when a considerable fraction of the observing time will be dedicated to long-term scientific key projects.

MPIfR in Bonn operates a 100-m telescope at Effelsberg. Following a fracturing of the azimuth track in 1993 and 1996, the track has been replaced and the telescope is now back in action with new azimuth wheel bearings, slightly changed wheel geometry, overhauled motors and new elevation encoder. During 
the last two years, several new or improved receiving systems have been installed at the secondary focus - new dual-feed $6-\mathrm{cm}$ receiver, new $11-\mathrm{cm}$ receiving system, new multi-frequency system containing dualchannel receivers at $2,1.3$ and $0.7 \mathrm{~cm}$ (for particular use in future VLBI activities), and a three-feed 9-mm system for continuum mapping.

The Green Bank Telescope (GBT), an offset paraboloid antenna with a reflector diameter of $100 \mathrm{~m} \mathrm{x}$ $110 \mathrm{~m}$, is under construction at NRAO, Green Bank (West Virginia). An unblocked-aperture design will increase the useful reflector area and eliminate reflections and diffraction effects which ordinarily complicate the response pattern of a conventional parabolic-reflector antenna. The 2000 reflecting panels will be mounted on actuators to facilitate surface adjustment. A laser-ranging system using a ring of ground stations surrounding the telescope will monitor the deformation of the reflecting surface under forces of gravity, wind, and temperature variation, enabling compensation by telescope motors, subreflector and panel actuators. Designed to operate at frequencies between $25 \mathrm{MHz}$ and $52 \mathrm{GHz}$, the expected final aperture efficiency at $50 \mathrm{GHz}$ is $60 \%$. It is hoped that high-quality observing will be possible even at frequencies as high as $80 \mathrm{GHz}$, with 3-arcsec pointing in calm conditions. The anticipated commencement of radio astronomy operation is 1998 . Several of the Gregorian focus front-end receiving systems have been completed and installed on the $140-\mathrm{ft}$ telescope for user evaluation. Work is continuing on the new $256 \mathrm{~K}$ digital spectrometer; it will provide instantaneous bandwidths of up to $800 \mathrm{MHz}$ on eight separate IF outputs.

The 76-m Lovell Telescope at Jodrell Bank continues to be used in Multi-Element Radio Linked Interferometer (MERLIN), VLBI, and for single-dish research, mainly involving pulsars. A major upgrade of the dish is planned, and preparations have included assessment of the foundations, azimuth track, and dish structure, and computer modelling of the elevation rotating structure.

The 64-m telescope of Parkes Observatory, part of the AT, was used by the SETI Institute for Project Phoenix during the first half of 1995. For the project, the Australia Telescope National Facility (ATNF) constructed a low-noise receiver and appropriate feed horns for operation in the frequency band 1.0-3.0 GHz. At the end of 1996, NASA will use the telescope to track the Galileo spacecraft orbiting Jupiter. To prepare for the tracking, a new prime focus cabin has been installed to enable a quick switching between receiving systems; the translator mechanism can support four standard receivers. A 21-cm multibeam receiver, consisting of a focal-plane array of 13 beams, is due to be commissioned in late 1996. It is intended principally for an extragalactic HI survey of the southern sky together with a deep HI search for optically obscured galaxies behind the southern galactic plane; however, a 20-cm survey for pulsars will be conducted simultaneously with the HI observations.

Major revisions have been made to the Cassegrain receiver system of NRAOs Green Bank 140-ft telescope. The maser-upconverter systems have been replaced by X-and K-band HFET receivers built for the GBT. This has resulted in performance and stability improvements, as well as providing experience in equipment to be used on the GBT. The receivers will be transferred to the GBT in 1997. The telescope is used for routine monitoring of frequencies of particular astrophysical interest, to both build up an interference data base and a list of relatively clear frequency bands. When the GBT begins operation, the telescope will cease to be supported as a general-user facility.

The Istituto di Radioastronomia has 32-m dishes in Medicina (Bologna) and Noto (Siricusa). The first operates in several frequency bands between 1.4 and $43 \mathrm{GHz}$; the second operates between 0.3 and $22 \mathrm{GHz}$. The antennas are used for VLBI, spectral-line observations, and flux-density monitoring.

The Hartebeesthoek Radio Astronomy Observatory (HartRAO) situated $60 \mathrm{~km}$ west of Johannesburg has a 26-m cassegrain antenna which operates at frequencies from $1.6 \mathrm{GHz}$ to $12 \mathrm{GHz}$. It is being used effectively as a single dish for surveys of $\mathrm{OH}$ and $\mathrm{CH}_{3} \mathrm{OH}$ masers and also plays an active role in southern hemisphere VLBI programs.

Shanghai Observatory operates the Shanghai VLBI Station, Sheshan. It has a 25-m radio telescope equipped with a VLBA data acquisition system and S2 recording terminal. In addition to VLBI, it is occasionally used for single-dish continuum and spectral-line observations. A new 22-GHz receiver, provided by the ATNF, was installed on the antenna during 1996, yielding a system temperature of about $100 \mathrm{~K}$.

Established in 1994 and operated by the Urumqi Astronomical Station, the Urumqi VLBI station has a 25-m radio telescope equipped with receivers operating at wavelengths of $92,18,13 / 3.6$, and 6 $\mathrm{cm}$, and a VLBI data acquisition system. A new $22-\mathrm{GHz}$ receiver provided by the National Astronomical 
Observatory of Japan (NAOJ), will be added towards the end of 1996. Although mainly used for VLBI observations, the telescope is occasionally used for pulsar and radio continuum observations.

The Owens Valley Radio Observatory (OVRO) of the California Institute of Technology (Caltech) is located at an altitude of $4000 \mathrm{ft}$ on the eastern side of the Sierra Nevadas near Bishop, California. It has several instruments operating at $\mathrm{cm}$ wavelengths. A $5.5-\mathrm{m}$ telescope has been in operation since 1993, providing fundamental measurements of the Cosmic Microwave Background (CMB) at $32 \mathrm{GHz}$, both in the detection of intrinsic anisotropies on angular scales of 7-20 arcmin and in the detection of the Sunyaev-Zeldovich effect (SZE) in nearby galaxy clusters. It provided the first detection of the SZE in a nearby cluster, and is currently observing the effect in an X-ray limited sample of clusters; these measurements will assist in reducing the systematic errors due to cluster elongation in the estimation of the Hubble Constant $\left(\mathrm{H}_{0}\right)$. The Observatory also has a $40-\mathrm{m}$ telescope operating in the frequency range 13-22 GHz. It is used in conjunction with the $5.5-\mathrm{m}$ telescope for CMB measurement; under-illuminated at $14.5 \mathrm{GHz}$ to match the $5.5-\mathrm{m}$ beam, it provides a second frequency channel for spectral discrimination. It is used at $20 \mathrm{GHz}$ for high-resolution driftscan mapping to enable point sources to be subtracted from the 5.5-m telescope data. With its smaller beam it complements the SZE project on the $5.5-\mathrm{m}$ telescope, and has provided early detections of the SZE in more distant clusters. It is also used independently for driftscan surveys of high-frequency radio sources, and for monitoring of recently discovered soft gammaray repeaters and new gravitational lenses.

Orbiting Telescopes. The next Swedish satellite ODIN will be an orbiting observatory devoted to astronomical and aeronomical spectroscopy at $\mathrm{mm}$ and sub-mm wavelengths. Onsala engineers are currently designing and building major components of the sub-mm receivers.

\subsubsection{Antenna Arrays}

Existing Sub-millimetre/Millimetre Wavelength Arrays. A sixth 10-m antenna has been added to the Nobeyama Millimeter Array (NMA). 230-GHz receivers have been installed on two antennas.

The Berkeley-Illinois-Maryland-Association (BIMA) mm-wave array at Hat Creek (California) is now an operational 9-element interferometer with baselines as large as $1.3 \mathrm{~km}$ providing a resolution of 0.4 arcsec at $3 \mathrm{~mm}$ wavelength. The array has an extremely flexible wideband correlator enabling observations of both the broadest-known mm-wave spectral lines in galaxies and the narrowest lines in quiescent molecular clouds. The array is capable of full remote operation, including receiver tuning, pointing, calibration, data gathering and data transfer. Quarter-wave plates have been recently installed to provide sequential switching between right and left circular polarization. This capability will enable the imaging of magnetic fields associated with warm dust in molecular clouds (in particular those containing observed Zeeman effects). Future developments include completion of outfitting with 1 -mm receivers, and adding a tenth antenna. The increased number of antennas will facilitate snapshot observations, and lessen the effects of atmospheric fluctuations. The scientific achievements of the current BIMA array include the highest resolution mm-wave observations using a single connected array, the first large mosaics with all spatial frequencies using a single instrument, and a sizeable number of surveys of galactic and extragalactic sources.

Berkeley researchers are investigating schemes for correcting atmospheric phase fluctuations based on measurements of the fluctuating atmosphere brightness in the total power of an antenna. For observations with the BIMA array, it was found that on a time-scale of several minutes, visibility phase fluctuations for one baseline were correlated with the difference in total power for the two antennas. Another possible approach is based on a rapid switching between target source and nearby phase calibrator source; Hat Creek results have shown that if the phase-calibration source is at intervals of only a few minutes, the largest atmospheric phase fluctuations can be partially removed by subtraction of the calibrator phase from the source phase.

The OVRO operates a mm-wave array, consisting of six 10.4-m antennas, in the frequency ranges 85-115 and 215-270 GHz. Currently, the antennas can be stationed with baselines of up to $220 \mathrm{~m} \mathrm{~N}-\mathrm{S}$ and $200 \mathrm{~m} \mathrm{E}-\mathrm{W}$; expansion of maximum baselines to $400 \mathrm{~m}$ in both directions is underway. Each antenna has $3-\mathrm{mm}$ and $1-\mathrm{mm}$ dual-channel SIS receivers feeding correlators with a combined width of $2 \mathrm{GHz}$. The highest angular resolution is 0.7 arcsec at the moment. The $3-\mathrm{mm}$ receivers have been recently outfitted with tuneable polarizers which can be set to any desired polarization state. The array has a fully automated observing system and remotely accessible archive database extending back to 1991 . The 
observing programs have involved spectral-line and continuum imaging of phenonema such as cometary and planetary atmospheres, stellar circumstellar envelopes, galactic high-mass star-formation regions, and features (nuclear distributions, central bars and spiral arm molecular-cloud concentrations) in nearby spiral galaxies. In addition, $\mathrm{CO}$ has been imaged with arcsec resolution in high redshift objects. Imaging the SZE in galaxy clusters observed at X-ray wavelengths has become possible with the installation of 26-GHz HEMT receivers on all antennas.

Several major steps have occurred in the development of the IRAM interferometer. During 1995, dual-channel $3-\mathrm{mm}$ and $1.3-\mathrm{mm}$ SIS receivers were added to the array. The receivers perform so well that 230-GHz total power measurements can be used for active phase corrections. The E-W track was extended, with the addition of three more stations, to a length of $408 \mathrm{~m}$, while the N-S track was extended with the addition of one more station to $232 \mathrm{~m}$. Sub-arcsec imaging is now possible. More recently, Antenna 5 has been completed and is now in operation.

Future Sub-millimetre/Millimetre Wavelength Arrays. The Submillimeter Wavelength Array (SMA) is under construction and is expected to begin reasonably routine operation by the end of 1999 on Mauna Kea near the Caltech Sub-millimetre Observatory (CSO) and JCMT. Initially, it will consist of six 6$m$ diameter antennas arranged on the sides of Reuleaux triangles. Four array configurations will be available with diameters of approximately $24,64,171$ and $470 \mathrm{~m}$. A cryostat at the Nasmyth focus of each antenna will accept eight receivers covering all the usable frequency bands between 230 and 850 $\mathrm{GHz}$. The maximum angular resolution will vary from 0.4 to 0.1 arcsec over this range. The array will have a special purpose XF correlator which will accept two channels (for either dual-polarization or dualfrequency operation) each of bandwidth $2 \mathrm{GHz}$. In the laboratory, the SIS mixer receivers with junctions fabricated at the US Jet Propulsion Laboratory (JPL) have yielded (DSB) receiver temperatures of 23, 30 and $65 \mathrm{~K}$ at 230,345 and $460 \mathrm{GHz}$. In June 1966, an agreement for collaboration was signed between the Smithsonian Astrophysical Observatory (SAO) and the Institute of Astronomy and Astrophysics of the Academy of Science in Taiwan (ASIAA). ASIAA will initially provide two additional antennas for the array and the associated electronics to increase the number of baselines from 15 to 28. Preparations are being made to include the 15-m JCMT and 10-m CSO telescope in the array on a part-time basis.

Several other large sub-mm or $\mathrm{mm}$ arrays are being proposed, and their completion should mark a new era for radio astronomy. NRO has proposed a new project, the Large Millimeter and Submillimeter Array (LMSA), with fifty 8-10 m antennas and baselines longer than $2 \mathrm{~km}$, high-quality imaging between $80 \mathrm{GHz}$ and $800 \mathrm{GHz}$. With a maximum baseline of $2 \mathrm{~km}$, an angular resolution of 0.2 arcsec would be achievable at $300 \mathrm{GHz}$.

NRAO has proposed the Millimeter Array (MMA), consisting of forty 8-m antennas erected on a site of high elevation, and providing operation in all atmospheric windows between $30 \mathrm{GHz}$ and $850 \mathrm{GHz}$ and baselines of $80 \mathrm{~m}$ to $10 \mathrm{~km}$ (with angular resolution as high as 10 mas). This project has evolved considerably over several years, and an initial design has now been submitted for development funding. Planning discussions for MMA partnerships with international groups and other government agencies are proceeding; a promising possibility of combining the MMA and LMSA periodically and thereby creating a single array with 10 mas angular resolution will be reviewed in 1997.

A European study project for a large $\mathrm{mm}$ array in the Southern Hemisphere (LSA) is continuing. A two-year activity involving IRAM, ESO, OSO and the Netherlands Foundation for Radio Astronomy (NFRA), the broad conceptual aims are a total collecting area of about $10,000 \mathrm{~cm}^{2}$ (e.g. $50-60 \times 15-$ $\mathrm{m}$ antennas), frequency range $43-350 \mathrm{GHz}$, and angular resolution of 0.1 arsec at $100 \mathrm{GHz}$. The study includes a search for an appropriate site in Chile.

Sites in northern Chile are being considered for the future $\mathrm{mm}$ arrays - the area contains one of the most arid deserts on Earth, with a mean annual precipitation below $10 \mathrm{~mm}$. The site-testing activities have been conducted in a collaboration between NRO, SEST/ESO, University of Chile, and NRAO. NRAO had previously considered other sites such as the Magdelena Mountains near Socorro (New Mexico) and the east coast of Mauna Kea (Hawaii). NRO is in fact also considering Mauna Kea (Hawaii) as a site for its array; this would make effective use of the infrastructure set up for the SUBARU 8-m telescope. However, because other groups are already site-testing this site, NRO decided to concentrate site-testing activities in Chile. Starting in 1992, NRO members visited about 20 possible sites, set up four meteorological stations, and used a $220-\mathrm{GHz}$ tipping radiometer developed at $\mathrm{NRO}$ to measure atmospheric opacities at five sites (including Paranal). In 1995, the radiometer and an $11-\mathrm{GHz}$ radio seeing monitor (RSM) were installed 
at the Rio Frio site (elevation $4050 \mathrm{~m}$ ). The RSM is an interferometer consisting of two 1.8-m antennas separated by about $300 \mathrm{~m}$, and receiving an $11.2-\mathrm{GHz}$ signal from an INTELSAT satellite. It provides direct measurements of the atmospheric phase fluctuations. NRAO also located similar equipment at the Chajnantor site, also called the San Pedro de Atacama site (elevation $5050 \mathrm{~m}$ ), about $300 \mathrm{~km}$ northeast of the other site. Analysis of the opacity and phase stability at the sites for more than six months indicates that both are excellent $\mathrm{mm}$ and sub-mm sites, but that Rio Frio has $225-\mathrm{GHz}$ opacities $(0.06$ and below for half the time) which are about $40 \%$ higher, and rms phase fluctuations (less than 4 deg for half the time) which are about $20 \%$ higher. For the Chajnanter site, the terrain significantly limits possible antenna configurations; however, with the aid of digital site elevation models, recent studies have shown that a $12-\mathrm{km}$ array can be accommodated.

Centimetre to Metre Wavelength Arrays. For the 27-element Very Large Array (VLA) of the NRAO, three more $40-50 \mathrm{GHz}$ receivers have been added to the ten built by NRAO with funds from the Institute of Astronomy of the Universidad Nacional Autonoma de Mexico. With retrofitting to replace first stage amplifiers in the previous receivers, the zenith system temperature is about $70 \mathrm{~K}$. A general VLA Upgrade Project is in progress. Design and prototyping is underway for a new generation of wide bandwidth receivers - all receivers except the 1.5-, 8- and 45-GHz systems will be replaced with modern HFETamplifier designs. In addition, the correlator will be replaced with one capable of wider bandwidths.

The Molonglo Synthesis Telescope (MOST) is operated by the University of Sydney (Australia) at a frequency of $843 \mathrm{MHz}$. A recent upgrading has increased the field size from 70 arcmin to 160 arcmin. This has been achieved without loss of sensitivity by installation of 352 HEMT (typically $12 \mathrm{~K}$ ) uncooled preamplifiers and computer-controlled phasing of the array to give time-domain multiplexing. For a complete 12-hour synthesis observation, the sensitivity is $1-2 \mathrm{mJy}^{\text {beam }}{ }^{-1}$ with a beamwidth of 43 arcsec.

The synthesis telescope of the Dominion Radio Astrophysical Observatory (DRAO), Penticton has both continuum (1420 MHz and $408 \mathrm{MHz})$ and spectral line (HI) capability. The angular resolution (1.0 and 3.5 arcmin) and wide field ( 2 and 8 degrees) at the two frequencies makes the telescope well suited for studies of the interstellar medium (ISM) of our Galaxy and nearby galaxies.

The Westerbork Synthesis Radio Telescope (WSRT) is undergoing an upgrade which began in 1995 and is expected to be complete in 1999. The antennas are undergoing extensive maintenance. A flexible pulsar filter bank has been added, enabling a tied-array mode of operation. Multi-frequency frontend (MFFE) receivers will be installed, providing wide bands in the frequency range $200-10,000 \mathrm{MHz}$. The backend correlator will be replaced by a correlator with high capacity in early 1997.

The OVRO has a 5-element solar array operating in bands in the frequency range 1-18 GHz. Three 2-m antennas augment the two 27-m antennas of the original Owens Valley $\mathrm{cm}$-wave interferometer. Designed to study magnetic fields at the surface of the Sun, the combined imaging and frequency agility provide not only flux density spectra of solar flares and active regions, but also brightness temperature spectra which can be compared directly with theory.

The Nancay Radio Observatory operates a radioheliograph dedicated to observations of the solar corona at frequencies between 150 and $450 \mathrm{MHz}$. It consists of 42 antennas each of diameter $4 \mathrm{~m}$, set in a T-shaped configuration of dimensions $3 \mathrm{~km}$ (east-west) $\times 1.2 \mathrm{~km}$ (north-south). During 1996 the northsouth branch will be lengthened to $2.3 \mathrm{~km}$ to provide a more circular beam to complement results being obtained by the SOHO spacecraft. The interferometer is currently being equipped with a new correlator to enable rapid (20 times per second) two-dimensional imaging at as many as ten frequencies.

The Nancay Radio Observatory also has a decametric array - the largest operational dual-polarity telescope for frequencies of $10-100 \mathrm{MHz}$. It is still mainly used for observations of the magneto-ionized environment of Jupiter and of the Sun. Recently, eruptive stars and pulsars were also detected with it. In 1995 the time resolution of the acousto-optical spectrometer was increased to $3 \mathrm{msec}$, enabling the detailed study of rapid Jovian radio bursts.

The Nobeyama Radioheliograph of NRO began routine observations at $17 \mathrm{GHz}$ in 1992 . Eighty-four $80-\mathrm{cm}$ antennas arranged in a $\mathrm{T}$-shaped array provide a spatial resolution of 10 arcsec and temporal resolution of $1 \mathrm{sec}$ or better. More than 1300 events have been imaged. Dual-frequency observation at 17 and $34 \mathrm{GHz}$ began in November 1995 .

The Australia Telescope Compact Array (ATCA) consists of a 6-km array of six 22-m antennas operating in bands at 20,13,6 and $3.5 \mathrm{~cm}$. Recent developments include provision of an on-line mosaicing 
mode, a tied-mode in which all antennas can be linked together in phase (e.g. for VLBI operation), a binning correlator mode enabling the collection of data in a set of time intervals synchronized to a periodic signal (e.g. for pulsar observations), and an option enabling position correction of the antennas using the phase reference calibrators during observing. A $225-\mathrm{GHz}$ water vapour radiometer has been constructed as a $\mathrm{PhD}$ project, spearheading future initiatives to correct for atmospheric phase errors at high frequencies.

All 45-m antennas of the Indian Giant Metre-wave Radio Telescope (GMRT), in which 12 antennas are located in a central array $1 \mathrm{~km} \times 1 \mathrm{~km}$ in size and 6 antennas are set along each of three 14-km arms of a Y-array, have been handed over to the Tata Institute for Fundamental Research (TIFR) by the contractors. Front-end electronics will be fitted to all antennas at $150,235,327$ and $610 \mathrm{MHz}$ by the end of 1996. Interferometer observations are being made for baseline calibration, and pulsars have been observed. Electronics for frequency ranges $40-60 \mathrm{MHz}$ and $1000-1430 \mathrm{MHz}$ will be added in 1997 . It is hoped to start regular observations by mid-1997.

Waseda University (Japan) has completed its 64-element direct-imaging digital interferometer, operating at $10.6 \mathrm{GHz}$. The HEMT receivers were installed during late 1995. Gamma-ray quasars (QSOs), Active Galactic Nuclei (AGNs) and galactic transient radio sources are being monitored.

The Istituto di Radioastronomia continues to operate its $408-\mathrm{MHz}$ transit instrument in Medicina (Bologna). It is a T-shaped array with $560-\mathrm{m}$ arms for which the east-west arm is a $560 \mathrm{~m} \mathrm{x} 25 \mathrm{~m}$ cylindrical parabola tiltable in declination, and the north-south arm contains 64 cylindrical-parabolic antennas $125 \times 8 \mathrm{~m}^{2}$. The instrument is mainly used for surveys, pulsar observations, and flux-density monitoring.

The Pushchino Radio Astronomy station has a new 151-MHz array consisting of 1344 nine-element Yagi antennas operating as a meridian instrument. It has a beam size of $0.5^{\circ}(\mathrm{EW})$ and $1^{\circ}$ (NS) and an effective area of $7000 \mathrm{~m}^{2}$. It has been used since mid-1995 for the monitoring of shock waves and other perturbations in interplanetary plasma. Simultaneous observations with 32 beams separated about one degree in declination provide daily maps of interplanetary scintillation indices.

The Square Kilometre Array. Amongst the concepts for future large-scale facilities in astronomy is the next-generation Square Kilometre (1KT) radio telescope. The purpose is to provide a facility with an order-of-magnitude increase in collecting area compared with that currently available. The requirement is based on the premise that significant new discoveries occur with each leap in effective collecting area. Groups in several countries (Australia, Canada, China, France, India and the Netherlands) are interested in constructing such a telescope operating in the $\mathrm{cm}$ to $\mathrm{m}$ wavelength range, and an URSI-IAU Working Group has been set up to coordinate the various interests. Although the project is science-driven, its realization will provide a great technological challenge. For instance, new concepts such as flat-tile antennas will require consideration. The Canadian concept has flat elements which could be tilted by actuators, whereas the Indian concept is based on the antenna philosophy of the GMRT.

\subsubsection{LBI and VLBI Networks}

Millimetre Wavelength Networks. A mm-VLBI array coordinated by the Haystack Observatory of the Massachusetts Institute of Technology (MIT) consists of antennas at Onsala, Bonn, Pico Valeta and Metsahovi in Europe, Haystack, Quabbin, Kitt Peak, Owens Valley in USA, Nobeyama in Japan, and SEST in Chile. Observing sessions at $86 \mathrm{GHz}$ are run on average every six months.

Combined 3-mm and 1.3-mm experiments have been carried out between the IRAM $30-\mathrm{m}$ telescope and one antenna of the IRAM interferometer.

Centimetre Wavelength Networks. The European VLBI Network (EVN) is a consortium of 14 institutes with 16 radio telescopes in Europe and Asia. It operates at wavelengths of $0.7,1.3,3.6,3.6 / 13,5,6,18$, 21, 50 and $90 \mathrm{~cm}$, either alone or in conjunction with the NRAO's Very Long Baseline Array (VLBA) and the MERLIN array. Comprehensive support for users is available at the Joint Institute for VLBI in Europe (JIVE). Recent significant developments include the first fringes for new antennas (e.g. the 32-m telescope in Torun, Poland), physical implementation of the MkIV upgrade, continuing construction of the 16-station data processor at JIVE, and organized assistance for new EVN participants (e.g. Urumqi, Shanghai and Torun). Efforts are being made to improve the present and future quality of EVN amplitude calibration data. 
The VLBA, consisting of ten 25-m antennas distributed across US territory, is now an operational instrument, performing routine astronomical observing $60 \%$ of the time. The array is now operated in nine frequency bands between 0.3 and $43 \mathrm{GHz}$; plans are in place to extend the range to $86 \mathrm{GHz}$. The VLBA correlator has more than sufficient capacity to keep up with the large amount of data generated by both the VLBA and global networks. The correlator software has undergone major revisions to produce a more robust and automated operation. The VLBA can now operate efficiently in conjunction with the Effelsberg 100-m and Green Bank 140-ft telescopes because observations and monitor data with these telescopes can now be written in VLBA-style format.

The MERLIN array is being upgraded to provide frequency flexibility. Three of the dishes, at Darnhall, Knockin and Pickmere, which are the same as VLA dishes, have had a rotating carousel installed at the secondary focus. The carousel can carry up to six receiving systems, and by rotation, any system can be placed on axis at the focus position within one minute. Dual-frequency operation at 5 and $22 \mathrm{GHz}$ will be offered in 1997, and a 1.4-GHz system will also be available the following year.

A Japanese domestic VLBI network (JNET) has been set up involving the NRO 45-m antenna, a 10-m antenna in Mizusawa, a 6-m antenna in Kagoshima, and often the 34-m antenna of the Communications Research Laboratory. A limited configuration of this network was effective in providing the first positional information for the separate water vapour velocity components in NGC 5248. Routine 22-GHz observing is not possible with the network.

For the Australian VLBI array, wideband S-2 terminals have been installed at the ATNF and Hobart antennas. The AT S-2 correlator has been expanded to 6 playback terminals for handling six stations, providing a rapid turn-around even during observing sessions. An international experiment between Tidbinbilla (Australia) and Bear Lakes (Russia) has been undertaken to compare MkII and S-2 b-factors. It is possible to use the S-2 terminals to directly record pulsar signals so that they can be played back and de-dispersed coherently rather than be de-dispersed via a band of filters. The $4^{\text {th }}$ Asia Pacific Telescope (APT) Workshop was hosted by ATNF in December 1995.

Space VLBI. The VLBI Space Observatory Programme (VSOP) is a joint project between the Institute of Space and Atmospheric Studies of Japan (ISAS) and NAOJ to launch an 8-m antenna into a 20,000 $\mathrm{km}$ high orbit. The ground-based infrastructure for VLBI measurements has been set up - antennas committed, observing programs selected. Launching scheduled for September 1996 has been delayed until February 1997.

The URSI/IAU Global VLBI Working Group (GVWG) has assisted in coordinating the ground arrays to be involved in VSOP. A special working group of the GVWG is working on the problem of scheduling ground-space VLBI observations.

VBLI Techniques and Recording Systems. Phase-referenced VLBI has proven to be an exceptionally powerful tool for the absolute astrometry of weak sources. Sub-milliarcsec precisions have been demonstrated for sources as weak as $2 \mathrm{mJy}$ during observations to link the Hipparchos optical star catalogue to the extragalactic radio reference frame using optically bright radio stars.. The phase-referencing technique is being further refined and applied to a VLBI search for planets orbiting nearby radio stars.

Radio Astrometry. VLBI astrometry of optically bright radio stars to link the Hipparchus optical star catalogue to the extragalactic radio reference frame was carried out over many years and has now been completed.

The measurement on the Digitized Sky Survey of the optical positions of 153 QSOs with accurate VLBI positions has shown that the rms uncertainty of the optical positions is 0.6 arcsec in both coordinates except for objects located near the edge of the Survey plates.

\subsection{NEW SITES FOR RADIO ASTRONOMY}

\subsubsection{Radio Astronomy from Antarctica}

The Antarctic Submillimeter Telescope and Remote Observatory (AST/RO), consisting of a 1.7-m offaxis parabola submillimeter telescope operating at 230 and $492 \mathrm{GHz}$, was installed at the Amundsen-Scott Station at the South Pole in late 1994. The telescope is primarily designed for a large-scale survey of the 492-GHz CI line in our Galaxy and the Magellanic Clouds. The receivers have SIS mixers and receiver 
temperatures around $200 \mathrm{~K}$ (DSB). Two accousto-optic spectrometers are available with bandwidths of $100 \mathrm{MHz}$ and $1 \mathrm{GHz}$. Observations have been on-going since early 1995, with studies of the large-scale distribution of the $\mathrm{CI}$ line in the galactic plane, the z-extent of the CI emission at selected longitudes, galactic HII regions, and galactic high-latitude clouds, and studies of the Large Magellanic Cloud. It has been found that the CI emission is spatially more extended than, and complementary to, the CO emission. Observations of molecules in the Earth's stratosphere have also been obtained. The project is a collaboration involving astronomers from SAO, the University of Illinois, Boston University, and the University of Colarado.

\subsubsection{Future Radio Astronomy from the Moon}

Because of the increasing use of the radio spectrum by transmitting services, radio astronomy is faced with the future prospect of increased interference to radio astronomy observations, and sites of low interference are being sought. A large area of the back side of the Moon viewed from the Earth is always hidden from the Earth and low-orbiting transmitters, and is thus shielded from terrestrial interference. There are already proposals to install VLF instrumentation in this shielded zone, together with international proposals to keep the zone free from transmitting services.

\subsection{THE SEARCH FOR EXTRA-TERRESTRIAL INTELLIGENCE}

Projects related to the search for extra-terrestrial intelligence (SETI) are taking place at several institutes around the world, and in general radio astronomy facilities are used, usually in conjunction with a veryhigh resolution spectrometer provided by the SETI researchers. This area of research is fully covered by IAU Commission 51 (Bioastronomy: search for extraterrestrial life), and will not be discussed in this Report.

\subsection{INSTRUMENTATION}

\subsubsection{Multi Beam Systems}

A 15-element focal plane array receiver (QUARRY) has been operating for the last four years on the 14-m telescope of the Five College Radio Astronomy Observatory (FCRAO). In the near future QUARRY will be replaced by the second Quabbin Observatory imaging array (SEQUOIA), a 32-pixel array covering the frequency range 85-115 GHz. The use of MMICs, the increased number of beams, and other improvements will enhance the telescopes mapping capability compared with the current system. The receiver temperatures will be better by more than a factor of two. It is expected that a 16-element version will be available for use early in 1997 .

A $4 \times 4$ focal-plane receiver array operating at $100 \mathrm{GHz}$ is being constructed for the OSO 20-m antenna; the first SIS receivers, operating at $4 \mathrm{~K}$, have been manufactured. For spectroscopy, each receiver will feed a 1024-channel digital spectrometer.

A 25-beam SIS focal plane array operating at $100 \mathrm{GHz}$ has been constructed for the NRO 45-m telescope.

A 13-beam focal-plane array operating at $21 \mathrm{~cm}$ is being constructed for the Parkes 64-m telescope. At least initially, it will be used for an HI survey of the southern sky, and a simultaneous 'piggyback' pulsar survey. It is a collaborative venture involving several institutes in Australia, together with Jodrell Bank and the University of Cardiff. CSIRO is providing the feed design and construction of focal-plane feed system, cryogenic package, IF system and digital correlator backend. Jodrell Bank is providing the 21-cm low-noise amplifiers. Commissioning is planned for late 1996.

\subsection{TECHNIQUES}

\subsubsection{Mosaicing}

BIMA researchers have been developing and testing a homogeneous array capability, which is the central design issue for the planned NRAO Millimeter Array. The strategy involves mosaic observations with the compact array during a normal 6-8 hour track, coupled with single-antenna observations with all array antennas mapping the same extended field. The technique has been used to map $\mathrm{C}^{18} \mathrm{O}$ in NGC 2024. A mosaic of 7 fields, the map contains all spatial frequencies from 8 arcsec to 4 arcmin, and includes both cross-correlation and auto-correlation data. 
Approximately $15 \%$ of the observing time on the ATCA is spent observing mosaics. To optimize efficiency and ease of use, a mosaicing mode is built into both the on-line observing and off-line reduction systems. For example, the on-line system allows a new pointing centre to be observed every 25 seconds, with only a few seconds of this time consumed by slewing and other overheads. The largest mosaic produced on the ATCA to date is a 1344 pointing-centre spectral-line observation of the Large Magellanic Cloud. Joint imaging and deconvolution of this data produced a $1997 \times 2230 \times 120$ pixel cube.

\subsubsection{Data Processing}

The AIPS++ project is a collaboration of several radio observatories (ATNF, BIMA, NFRA and NRAO are currently actively involved) to develop the next generation of data reduction software. It specifically aims to provide general formalisms that apply to all observatories. Over the last three years the project has developed to a stage where real astronomical data can now be processed. Currently, the single-dish side of AIPS++ is used in the commissioning tests for the GBT and will also be used for the Parkes Multi-beam HI survey. In the synthesis-processing area, important progress has been made during the last year, including the completion of a full polarization imaging formalism for a generic interferometer. This has now been used to produce a number of good quality polarization images from VLA and WSRT data. AIPS++ will feature a programmable command-line interface supplemented by graphical user interfaces and a large $\mathrm{C}++$ library of general and astronomical classes. The first public release of AIPS ++ is planned for mid-1997.

\subsubsection{Laboratory Molecular Spectroscopy}

Japanese scientists have carried out extensive laboratory spectroscopic studies of a number of molecules and isotopomers of astrophysical interest such as $\mathrm{MgNC}, \mathrm{FeCO}, \mathrm{HCS}^{+}, \mathrm{CH}_{2}, \mathrm{CaNC}, \mathrm{AlH}$ and $\mathrm{ND}$. $\mathrm{MgNC}$ was subsequently detected in IRC +10216 .

\section{New Observational Radio Astronomy}

\subsection{OUR GALAXY}

\subsubsection{Solar Neighbourhood}

Solar Radio Astronomy. Expansion of the BIMA array to long baselines has led to the first arcsecondresolution images of solar flares, and images have been made of the impulsive non-thermal mm-wave emission for two flares. For a flare of 1994 August 16, comparison of the temporal behaviour of the emission with hard X-ray emission detected by the Compton Gamma Ray Observatory (GRO) of NASA showed two radio components. The first was a brief pulse at the onset of the $\mathrm{X}$-ray emission, whereas the second was of longer duration and caused by nonthermal electrons producing no X-rays. Such comparisons will provide important constraints for models of the spatial distribution of accelerated electrons in flares.

The Nobeyama Radioheliograph has detected many interesting phenomena which have been studied in combination with X-ray data from the YOHKOH satellite. Radio bright spots in the quiet Sun were found to have X-ray counterparts. Polarized gyroresonance emission was detected from a large sunspot. A prominence eruption and its following flare were studied with simultaneous optical, radio and X-ray observations, the results supporting the scenario that the reconnection of anti-parallel magnetic fields is produced by the prominence eruption. The high spatial and temporal resolution of the instrument have yielded many new features of flares.

The National Research Council of Canada has been making regular determinations of the solar flux at $10.7 \mathrm{~cm}$ wavelength since 1946 . This wavelength is close to the peak in the spectrum of the slowly varying solar component. These measurements have been adopted worldwide as the primary index of solar activity. Since 1990, the program has been operated from DRAO, using its 26-m antenna; data are distributed automatically to scientific and commercial users.

Solar Wind, Asteroids, Comets. Distributions of scintillation index in the interior region of an interplanetary shock wave have been constructed from 102.5-MHz imaging with the Pushchino Large Phased Array. The derived shock-front parameters correspond to distances of about $1 \mathrm{AU}$ from the Sun.

The velocity structure and plasma turbulence in the solar wind have been investigated at Kashima by observing interstellar scintillation of QSOs at 2,8 and $22 \mathrm{GHz}$. Ooty facilities and the GMRT have been used for simultaneous observations of the solar wind at high latitudes. 
In 1995, the 34-m Kashima telescope was used for radar observations of the Earth-approaching asteroid 6489 .

The IRAM 30-m telescope has been used for molecular-line observations of comets. $\mathrm{HCN}, \mathrm{H}_{2} \mathrm{~S}, \mathrm{H}_{2} \mathrm{CO}$ and $\mathrm{CH}_{3} \mathrm{OH}$ were detected in comet $109 \mathrm{P} / \mathrm{Swift}$-Tuttle; their strongly asymmetrical line profiles were probably due to the effects of jets seen at visual wavelengths. The $\mathrm{CO} \mathrm{J}=2-1$ line was detected in comet Hale-Bopp in 1995 and is now being monitored with the IRAM telescope and the JCMT. The line is slightly blue-shifted, indicating outgassing from the day side of the nucleus, which can be related to dust jets in optical images.

The QUARRY system on the FCRAO 14-m telescope was used in early 1996 to search for molecular lines in Comet Hyakutake. HCN J=1-0 emission was monitored; observed HCN structures were in good agreement with jet features present in images of the inner corona.

For other comets: several molecular-lines have been observed in comet $\mathrm{P} / \mathrm{Halley}-\mathrm{H}_{2} \mathrm{~S}, \mathrm{CH}_{3} \mathrm{OH}, \mathrm{H}_{2} \mathrm{O}$, $\mathrm{NH}_{3}$. The water vapour has yielded $\mathrm{D} / \mathrm{H}$ and ${ }^{18} \mathrm{O} /{ }^{16} \mathrm{O}$ abundance ratios. $\mathrm{CH}_{3} \mathrm{OH}$ has also been observed in comets Austin $1990 \mathrm{~V}$ and Levy $1990 \mathrm{XX}$

Planets. A global network of eleven radio telescopes observed Jupiter and the behaviour of its synchrotron and thermal radio emission before, during, and after the period of impacts of Comet ShoemakerLevy 9 in July 1994. Single dishes monitored the total radiation output on short time scales, while imaging arrays - VLA, WSRT and ATCA - were used for high-resolution imaging. The radio emission increased dramatically during the impacts; the change was wavelength dependent, ranging from around $10 \%$ at $70-90 \mathrm{~cm}$ to about $45 \%$ at $3.5-6 \mathrm{~cm}$. High-frequency VLA and ATCA observations showed that the thermal disk remained unaltered by the impact, whereas the non-thermal radiation and polarization from the Jovian Van Allen belts increased; the enhancement was localized in longitude and concentrated near the equator. However, dips in thermal emission at $0.8-3.6 \mathrm{~cm}$ were also reported. The radio spectrum hardened, flattening towards shorter wavelengths, during and after the impact. After the impacts, the flux density began to decrease at all wavelengths. ATCA observations twelve months later showed that the radiation has returned to its pre-impact state. There have been several suggestions for the cause of the synchrotron enhancement - acceleration of magnetospheric electrons by shock waves from the impact point; pitch angle scattering; an increase in the inward radial diffusion of electrons. On the basis of ATCA observations, a 3-D tomographic image of the radiation belts has been reconstructed. Characteristics of the deduced magnetic field close to the planet, fed into existing magnetic field models, appear to account entirely for the observed features of the radiation belts. The collision sites have been monitored with the JCMT in sub-mm spectral lines of CO and HCN for more than a year. The lines are seen in both emission and absorption, and vary with time.

The complete polarization state, as a function of both frequency and time, has been determined for a large number of Io-related and non-related radio storms observed with the spectro-polarimeter at Nancay Radio Observatory.

A joint study of Mercury using BIMA array and VLA data has shown that the planet's surface is largely devoid of basalt.

Four large main-belt asteroids (1 Ceres, 2 Pallas, 4 Vesta, 10 Hygiea) were detected with the BIMA array in 1995.

\subsubsection{Beyond the Solar System}

Stellar Radio Emission. The relatively new subject of radio stars has come of age in the last few years. Radio emission is being detected from a variety of stellar objects and their associated winds, outflows, disks and shells.

The new MERLIN, with 50- milliarsec resolution and 50-micro Jy sensitivity, has enabled researchers to resolve classical novae just 80 days after outburst and find asymmetries and brightness temperatures of $40000 \mathrm{~K}$. Other results include the detection of non-thermal emission and bipolar outflow in symbiotic stars. VLBI observations of Cyg X-3 show that the strong radio outbursts are accompanied by twin radio components which move out at around $0.3 \mathrm{c}$, whereas some weaker events may be associated with apparent superluminal brightening of an extended component (probably as a shell is illuminated). Millimeter-wave observations show that the ejected material moves through a dense wind which delays the onset of long wavelength radio radiation. 
Centimetre radio continuum has been detected from a few dozen low-mass pre-main sequence stars, or T-Tauri stars. Most objects are weak-lined T-Tauri stars, where the circumstellar disk is no longer interacting with the young star. Radio power ranges from $10^{16}-10^{18} \mathrm{~W} \mathrm{~Hz}^{-1}$, and flares with durations of hours are sometimes observed. These properties are similar to those of the RS CVn binary systems. The emission is interpreted as enhanced solar-type gyrosynchrotron radiation arising from the rapid rotation and convective interiors of stars descending the Hayashi tracks. Gyrosynchrotron emission has been directly confirmed in a few cases (Hubble 4, T-Tau South, HD 283447) where circular polarization is detected. In particular, HD 283447 is an unusually active young triple-star system that exhibits timevariable linear and circular polarization.

Radio emission at 8.6 and $4.8 \mathrm{GHz}$ has been detected in a sample of early-type stars (including WolfRayet stars, B hypergiants, Of stars and a luminous blue variable) using the ATCA. The radio spectrum between these frequencies is consistent with thermal emission from an optically thick wind. Thermal radiation has also been detected from the winds of the well-known blue variables AG Car, HR Car, He3-519 and WRA 751. An unexpectedly high mass-loss rate was required to account for the $8.6-\mathrm{GHz}$ intensity of the massive $\mathrm{O} 3 \mathrm{~V}(\mathrm{f})$ star $\mathrm{HD} 93250$, leading to the speculation that most of the emission at this frequency is non-thermal.

When first imaged with the ATCA at $3 \mathrm{~cm}$ in 1992, $\eta$ Car appeared as a simple star-like source with a flux density $(0.8 \mathrm{Jy})$ similar to that found a few months earlier. However, by June 1993 , twelve months later, it had developed a complex image with a total flux density of $1.2 \mathrm{Jy}$, with broad $(250 \mathrm{~km}$ $\mathrm{s}^{-1}$ ) recombination-line emission. Monitored regularly since then, the flux density peaked at $2.9 \mathrm{Jy}$ in November and December 1995, and is now decreasing. Recent imaging at both 3 and $6 \mathrm{~cm}$ reveals three main source components. One component is associated with $6-\mathrm{cm}$ recombination line absorption, and is believed to be a foreground gas cloud. On the grounds of both morphology and comparison with HST and other optical images, the other components appear to arise in an equatorial accretion disk and polar wind respectively.

Centimetre-wavelength emission from nearby long-period (Mira and semi-regular) variable stars has been detected with the VLA. Simple modelling with a single set of physical parameters can approximately fit the results, as well as providing plausible sizes and brightness temperatures at far-IR wavelengths. Distance estimates with a precision of about $10 \%$ may be possible.

Circumstellar Envelopes. Searches for more OH/IR stars continue. The $1612 \mathrm{MHz}$ OH survey of colourselected IRAS objects with the Arecibo 1000 -ft telescope was extended to objects with IR colours outside the original coverage, resulting in further detections. Some research with $\mathrm{OH} / \mathrm{IR}$ stars involve their galactic distribution. A dynamical comparison of two samples of these objects, one very close to the Galactic Centre, the other in the bulge and disk, show two distinct populations. One extends over the whole galaxy, showing signs of gradual evolution, whereas the other is found near the Galactic Centre, with high rotation and no signs of long evolution. A $43-\mathrm{GHz}$ SiO survey of IRAS sources has been used to investigate the kinematics in the galactic bulge. A systematic difference between the radial velocities of northern and southern objects has been attributed to a 7-degree tilt of the rotation plane of the bulge relative to the galactic longitudinal circle.

Circumstellar envelopes of late-type stars are often rich in molecular lines. The carbon star IRC +10216 has been the target of many studies, providing a rich harvest of different molecules. Highresolution BIMA molecular-line observations show shell-like distributions of some molecules in the extended envelope surrounding the star. The peak abundance of long carbon-chain molecules such as $\mathrm{HC}_{3} \mathrm{~N}$ was found to be very high compared with predictions based on early models of circumstellar chemistry. However, newer calculations which included neutral molecule-radical reactions show that the synthesis of relatively complex organic species could reproduce the observed abundances. A program of mapping the most significant molecules is underway with the IRAM interferometer, with short-spacing data provided by the IRAM $30-\mathrm{m}$ telescope. The results support the predictions of gas-phase models: radicals and cyanopolynes have hollow-shell distributions whereas small stable molecules are centrally peaked. Transitions of metal-containing molecules $\mathrm{NaCN}$ and $\mathrm{MgCN}$ have been detected in the outer envelope of the object with the NRAO $12-\mathrm{m}$ or IRAM $30-\mathrm{m}$ telescopes. Ten lines with harmonically related frequencies (three detected in a 3-mm survey with the IRAM 30-m telescope, and seven in a 7-mm survey with the NRO 45-m telescope) have been identified with $\mathrm{C}_{8} \mathrm{H}$. The NRO $45-\mathrm{m}$ telescope has been used for a 
spectral-line search in the frequency range $28-50 \mathrm{GHz}$. The CSO carried out a similar search, but in the range $330-358 \mathrm{GHz} ; 56$ lines were detected and related to 8 molecules and 18 isotopomers.

The NRO 45-m telescope and NMA have been used for $\mathrm{CO}, \mathrm{HCO}^{+}, \mathrm{HCN}$ and $\mathrm{SiO}$ studies of the circumstellar envelopes of several objects, including NGC 7027, IRAS 21282+5050, CRL 618, CRL 2688, CRL 3608, S Sct and other carbon-rich objects. The distribution of the emission was found to be almost circular. A possible relationship between chemical composition and evolutionary stage of the central star has been suggested.

The carbon star U Cam has been mapped in NHC and CN with the IRAM interferometer. A twoenvelope structure - outer envelope with radius of $7 \times 10^{16} \mathrm{~cm}$ and expansion velocity of $25 \mathrm{~km} \mathrm{~s}^{-1}$ surrounding inner envelope or radius $6 \times 10^{15} \mathrm{~cm}$ and expansion velocity of $13 \mathrm{~km} \mathrm{~s}^{-1}$ provides evidence of a significant mass loss ratio variation within the last thousand years.

The BIMA array has been used in a study of mass loss in three carbon-rich evolved stars by imaging the associated CO emission. IRAS $21282+5050$ (a planetary nebula) showed a core-halo structure consistent with episodic mass loss.

A systematic study of sub-mm $\mathrm{H}_{2} \mathrm{O}$ masers in circumstellar envelopes has been made using JCMT, with near-simultaneous observations of the 22- $\mathrm{GHz} \mathrm{H}_{2} \mathrm{O}$ line using telescopes of the Nuffield Radio Astronomy Laboratories (NRAL). Variability was observed in each line, but variations in the different transitions were often uncorrelated. Maser luminosity was found to increase systematically with mass-loss rate of the evolved star. In a similar study using a set of $382 \mathrm{OH} / \mathrm{IR}$ stars identified from colour-selected IRAS sources, maser emission was detected from half the sample; the $\mathrm{H}_{2} \mathrm{O}$ outflow velocities were on average 75 per cent of the $\mathrm{OH}$ circumstellar expansion velocities.

Circumstellar 22- $\mathrm{GHz} \mathrm{H}_{2} \mathrm{O}$ masers have been resolved using MERLIN. Brightness temperatures, measured for the first time, are close to theoretically predicted values. Proper motions of the masers have been made for the first time. The masers were found to move at the overall outflow velocity, indicating mass-loss in discrete blobs.

VLBA images of $\mathrm{H}_{2} \mathrm{O}$ and $\mathrm{SiO}$ masers in the envelopes of evolved $\mathrm{M}$-type giants have been made at four epochs over three years. In five objects, $\mathrm{H}_{2} \mathrm{O}$ masers lie systematically outside the $\mathrm{SiO}$ masers, in accordance with predictions. The $\mathrm{H}_{2} \mathrm{O}$ masers move outwards with transverse velocities more or less consistent with single-dish outflow measurements, enabling distances accurate to within $10 \%$ to be detemined by the cluster-parallax method.

Protoplanetary Disks. Several protoplanetary disks have been detected in the Taurus regions. Observations with the BIMA array have provided the first $\mathrm{mm}$ sub-arcsecond image of the disk around solar-mass star HL Tau. The disk has a semi-major axis of $70+/-15$ AU for a distance of $140 \mathrm{pc}$. The $2.7-\mathrm{cm} \mathrm{im-}$ age, earlier $0.87-\mathrm{mm}$ data, and intensity measurements between 10 microns and $1.3 \mathrm{~cm}$ have enabled the surface density distribution within a circumstellar disk to be constrained for the first time.

CO J=1-0 observations of T Tauri stars GG Tau and DM Tau with the NRO 45-m telescope revealed double-peaked emission features that were interpreted in terms of circumstellar disks. Follow up CO observations with the NMA confirmed the presence of rotating circumstellar gas disks of radius 500 and $350 \mathrm{AU}$ respectively. In addition, the gas around DG Tau was found to have a disk-like structure, with major axis perpendicular to the blue-shifted optical jet ejected from the star.

$\mathrm{CO}$ and $2.7-\mathrm{mm}$ continuum observations of young star systems have been made with the IRAM interferometer. Dust emissivity laws could be fitted to the $\mathrm{mm}$ spectral energy distributions. New images have been obtained of GM Aur, a pre-main sequence T Tauri star at a distance of $150 \mathrm{pc}$; the image shows thermal emission from dust in a circumstellar disk.

$\mathrm{CS} \mathrm{J=2-1}$ observations of $\mathrm{HH} 83$ have shown that the CS is concentrated in a bar-like feature and two ridges. The results were interpreted in terms of a rotating disk around a jet associated with the object.

In a molecular-line study of the protobinary source IRAS 16293-2422, 265 lines of 44 molecules and isotopomers have been detected in 230 - and $345-\mathrm{GHz}$ spectral windows. Three physically and chemically different components were identified: a warm and dense gas rich in organic molecules, of extent 3-10 arsec and probably representing the bipolar outflow with the circumbinary envelope; the circumbinary envelope itself, containing the more common molecules; a colder lower-density outer part of the envelope which gradually merges with the ambient surrounding cloud. These regimes are qualitatively similar to those found in high-mass star-forming regions. 
Pulsars. Pulsars are rare, extreme astrophysical objects, accurate clocks and excellent tools for tests of fundamental physics. Recent searches covering large areas of the sky have greatly increased the number of known millisecond pulsars in the galactic disk. The known population has increased from four in 1990 to 35 at present. These pulsars are important, not only because many of them are interesting in their own right, with for example eclipsing behaviour of complex pulse profiles, but also because they form a network of very precise clocks. Many applications of pulsar timing, for example detection of gravitational waves and establishment of a long-term standard of time, require many of these pulsars distributed across the celestial sphere. The survey with the Parkes telescope, a collaboration between the ATNF and Jodrell Bank, yielded 17 new millisecond pulsars, doubling the number known in the disk. Searches at other observatories, Arecibo, Jodrell Bank, Green Bank and Westerbork, have (collectively) found a similar number. The pulsar PSR J0437-4715, found in the Parkes survey, is by far the nearest and brightest millisecond pulsar known. Most of the new pulsars are binary, and several of them have an optically identified white dwarf companion. An NRAO 140 - $\mathrm{ft}$ survey for millisecond pulsars resulted in the detection of J1518+4904, a tight double-neutron-star binary system. Such systems can provide unique opportunities for measuring phenomena of relativistic gravity.

Regular timing of recently discovered young pulsars has resulted in the discovery of 25 glitches in 10 pulsars. This enabled the first statistical assessment and the rejection of crust cracking as the cause of most glitches.

Continued timing of PSR B1257+12 has revealed the effects of gravitational interaction between the two planets, confirming that the period variations observed in this pulsar are due to planet-sized bodies orbiting the pulsar. They also showed the presence of a third moon-sized planet in the system. This system remains the only detected pulsar with planetary companions.

The two known pulsars with massive, non-degenerate companions, PSR B1259-63 and PSR J00457319 , have both produced interesting results. When PSR B1259-63 passed through its second observed periastron in January, 1994, it became an X-ray and radio continuum source, probably due to synchrotron emission at a bow-shock interface with the Be star circumstellar disk. The pulsar, normally highly linearly polarized, lost all of its polarization about 100 days before periastron at $1.4 \mathrm{GHz}$ and later at higher frequencies. Both the rotation and dispersion measures changed dramatically, and the pulse became highly scattered as the pulsar passed through the circumstellar disk.

The effects of precession of the orbital plane of PSR J0045-7319 were detected in timing observations. The precession is attributed to coupling between the spin of the B-star companion and the orbital motion of the pulsar, the first time such spin-orbit coupling has been detected outside the Solar System. Detection of the effect also gives strong support to the idea that neutron stars receive a strong kick at birth. Such a kick is commonly invoked to explain the very high velocities observed for most pulsars. Recent proper motion measurements, coupled with a new model for the distribution of dispersing electrons in the Galaxy and consideration of selection effects, show that the median space velocity of pulsars is larger than previously thought, about $500 \mathrm{~km} / \mathrm{s}$. This has been interpreted as good evidence for asymmetric supernovae.

Although millisecond pulsars generally have smaller velocities than 'normal' pulsars, their transverse velocity is often large enough to contribute significantly to the observed period derivative through the 'Shklovskii' effect or secular acceleration. This raises the already great age of these pulsars, with several having an upper limit on their age greater than a Hubble time.

The Vela pulsar may also be older than previously thought. Analysis of 25 years of timing data suggests that the braking index may be about 1.4, much less than the 'magnetic-dipole' value of 3.0. This implies that the age of the pulsar may be up to four times the normally quoted characteristic age of 11,000 years. An increased age for the pulsar would affect interpretation of the X-ray and radio emission from the supernova remnant. A deconvolution technique has been applied to observations obtained with a VLBI network and the VLBA to yield the first image of a pulsar's radiosphere.

As part of a survey using the Arecibo $1000-\mathrm{ft}$ telescope at $430 \mathrm{MHz}$, timing measurements showed that PSR J2229+2643 has one of the smallest period derivatives detected, and the pulsar can be used to establish a new upper limit for the possible rate of change of the Newtonian constant of gravity.

The Pushchino Large Phased Array has been involved in low-frequency observations of pulsars. Analysis of timing data taken over many years for PSR B0329+54 has revealed a quasi-sinusoidal modulation of period 16.9 years. This periodicity could be interpreted as evidence of an orbiting planet-like body. PSR J0034-0534, one of the shortest millisecond pulsars with a period of $1.88 \mathrm{msec}$, has been detected at 
$103 \mathrm{MHz}$ (with a flux density of $0.6 \mathrm{Jy}$ ). Its integrated pulse width was less than at $430 \mathrm{MHz}$, and this was attributed to magnetic field distortion by the fast rotation of the pulsar magnetosphere. The spatial structure in the magnetosphere of several pulsars has been investigated from interstellar scintillations of 103-MHz pulsar radio emission.

A 1.5-GHz search with the Parkes 64-m telescope for pulsars in the Galactic Centre region failed to reveal any, confirming either that there is a lack of pulsars there, or that scattering is severe in the inner $100 \mathrm{pc}$.

The MOST has been used in gating mode for observations of the faint wind nebula associated with the Vela pulsar. A program is in place to observe the binary system PSR 1259-63 and its companion star SS 2883 at the next pulsar periastron in July 1997.

An 85- $\mathrm{ft}$ telescope at Green Bank is being used for frequent sampling (almost daily) of pulsars at 327 and $610 \mathrm{MHz}$; the goals include timing glitches, ephemeris for CGRO and XTE observations, refractive interstellar scintillation, and rotation and scattering measures. Other groups are, or plan to be, involved in frequent pulsar sampling e.g. groups in Hobart, Hartebeesthoek and Green Bank (Vela pulsar), Jodrell Bank (Crab pulsar), group in Torun (many pulsars).

An on-going pulsar VLBI project between Kashima and Kalyazin (Russia) commenced in 1995.

Planetary Nebulae. Studies of planetary nebulae continue, with specific interest in continuum observations of northern hemisphere candidates, and identification of distant objects at low galactic latitude.

Spectral-line studies have included VLA detection of circumnebular HI in SwSt 1, M 3-35 and perhaps $\mathrm{Hu} 2-1$. CO observations with the JCMT have revealed $\mathrm{J}=1-0$ or $\mathrm{J}=2-1$ emission associated with IC 5117, M 1-16 and NGC 6302. H92 $\alpha$ emission has also been detected in IC 5117. For M 1-16, a CO map shows that the molecular envelope contains both slow and fast outflows; high-excitation species such as $\mathrm{HCN}, \mathrm{CN}$, and $\mathrm{HCO}^{+}$have been found in the envelope. The dissociation and subsequent ionization of molecular material in a detached spherically symmetric stellar wind has been modelled as a star evolves into a planetary nebula.

Significant advances have taken place on radio observations of several planetary nebulae with the VLA at two separate epochs. These have yielded difference maps indicating the angular expansion of these objects. Combination with Doppler expansions has resulted in expansion distances which are accurate at the $20-30 \%$ level - a vast improvement over previous methods. Typical angular expansions for these objects are a few mas per year. Planetary nebulae with distances estimated by this method include NGC 3242, NGC 6210, NGC 6302, NGC 6572, BD30+3639, NGC 7027, and NGC 7662.

Galactic Superluminal Sources. The VLA and the NRT featured in the first detection of a galactic superluminal source, the strong X-ray source GRS $1915+105$, with five months of monitoring at several frequencies. The object underwent a radio outburst in 1994, revealing a pair of condensations with very large proper motions in its radio jets. After correction for relativistic effects, the apparent velocity of the condensation in the approaching jet was $0.92 \mathrm{c}$. Millimetre observations were consistent with the source being at a kinematic distance of $12.5+/-1.5 \mathrm{kpc}$ from the Sun.

Superluminal jets from another galactic object, the X-ray nova GRO1655, were detected with the VLBA. and tracked with a VLBA/VLA combination. Highly collimated relativistic jets each side of the central source expand and decay over a few days. The jet ejection, at $0.92 \mathrm{c}$, appears episodic and asymmetric. Optical observations suggest that the system is an eclipsing spectroscopic binary, with a black hole primary.

A MOST program is underway to monitor the $843-\mathrm{MHz}$ emission from X-ray transients. Objects of current interest are GRO1655, GRS1915+105, GX3 339-4, and GRS1716.

It has been suggested that superluminal galactic sources and other galactic radio jet objects such as 1E1740-2942, GRS 1758-258 and SS 433 form the framework of a jet/disk model developed initially for AGNs. There is an indication of a similar radio loud/radio weak dichotomy as found earlier for QSO radio cores.

Supernova Remnants. The properties of galactic supernova remnants (SNRs) continue to be investigated. Specific areas of interest include searches for new SNR identifications, comparison of multiwavelength morphologies, polarization, interaction with surrounding ISM, and searches for associated pulsars. 
A MOST survey of the southern galactic plane has been used to produce a catalogue of known and new SNR identifications. The objects show a wide range of morphologies, and the interactions of the SNRs with their surrounding ISM is under investigation. For the Vela SNR, X-ray and optical images are also being used in a multi-frequency study of the interaction between neutral and ionized gas. $2.4-\mathrm{GHz}$ observations of this SNR, some $8^{\circ}$ in diameter, with the Parkes 64-m telescope revealed several associated features extending beyond the general SNR shell.

The SNR group at the Institute of Astronomy and Space Physics (Buenos Aires), in collaboration with other researchers, have observed the radio continuum and spectral-line emission of SNRs with several telescopes. VLA observations, mostly at $1.4 \mathrm{GHz}$ but some also at $0.3 \mathrm{GHz}$, have been obtained for several galactic SNRs. For W44, an associated synchrotron nebula powered by pulsar PSR $1853+01$ was discovered. HI observations were also obtained for Pup-A, revealing the presence of colliding clouds. HI was also observed around $3 \mathrm{C} 400.2$, but with the DRAO telescope. Current projects include a mosaic of $\mathrm{W} 50$, and investigation of the expansion of $3 \mathrm{C} 10$.

$1720-\mathrm{MHz} \mathrm{OH}$ maser emission was detected around twenty SNRs with both the Green Bank 140-ft telescope and the Parkes 64-m telescope. Follow-up observations with the VLA and ATCA resulted in the detection of compact emission from 8 SNRs. The masers appear to be collisionally excited by the passage of the SNR shock through a molecular cloud.

In other observations, the Parkes telescope has been used to survey large HI fields around the Vela SNR, G18.8+0.3 and W 28. MOST observations of candidate SNRs G358.4-1.9 and G2.4+1.4 suggest a thermal origin for the radio emission.

Galactic Plane Continuum Surveys The MOST has been used for a new 843-MHz survey of the galactic plane in the longitude range $335^{\circ}-5^{\circ}$, and galactic latitudes within $+/-2^{\circ} .5$. The resolution is 90 arcsec $\mathrm{x} 43.5$ arcsec, and flux density lower limit $5 \mathrm{mJy}^{\text {beam }}{ }^{-1}$. Primarily on the basis of a separation between thermal and non-thermal emission, seventeen objects have been identified as new potential SNRs. A second-epoch survey is planned to investigate radio variability of sources. MOST observations of a six square degree at $l=312^{\circ}, b=0^{\circ}$ show an abundance of low-surface-brightness extended structure. This may be linked to the extended HII envelopes that have been postulated to explain certain galactic phenomena, or may represent an intermediate state between classical HIl regions and the warm ionized medium.

The 64-m Parkes telescope has been used for a 2.4-GHz polarimetric continuum survey of the southern galactic plane at longitudes between $238^{\circ}$ and $5^{\circ}$ and latitudes within $+/-9^{\circ}$; the lower intensity limits for continuum and polarized emission are 12 and $4 \mathrm{mJy} \mathrm{beam}^{-1}$. The images reveal a large amount of structure and detail, including a considerable number of low-surface loops and spurs.

A consortium of 33 scientists from Canada and other countries is using the DRAO Synthesis Telescope for a galactic plane survey, consisting of imaging of the continuum and HI emission in a strip 9.3 degrees wide along the plane between longitudes $75^{\circ}$ and $145^{\circ}$. The observations will be complemented by observations at other wavelengths, provided by international partners in the project, in particular $\mathrm{CO}$ images from FCRAO, high-resolution IR data from further computer processing of IRAS data (IPACJPL), 151-MHz continuum data from the University of Cambridge, and 232- and 327-MHz data from the Beijing Astronomical Observatory. The DRAO survey observations began in 1995 and will occupy most of the time of the Telescope until the year 2000. The data will be made public beginning in 1988 through the Canadian Astronomical Data Centre in Victoria. One of the first results is the discovery of a galactic chimney, associated with the HII region W4 (IC 1805) in the Perseus Arm. The combined winds of a cluster of nine O-type stars have blown out through the galactic disk, an example of a conduit for replenishment of the galactic halo from the disk.

Interstellar Medium. Tiny-scale atomic structure (TSAS), on scales of tens of astronomical units, was first detected twenty years ago using HI VLBI techniques; it was not until fourteen years later that the discovery was confirmed. TSAS has now been detected by time-variable HI absorption towards pulsars, caused by the moving pulsar sweeping its line-of-sight over the structure. In fact TSAS has been seen against every source towards which it has been sought. It has also been detected with angle-variable optical absorption lines against a sample of closely-spaced stars. The TSAS is ubiquitous within the ordinary cold neutral medium (CNM). The phenomenon is puzzling because the implied pressures are some 44 times larger than the hydrostatic pressure on the galactic plane, and 300 times larger than the 
standard CNM thermal pressure. This is unacceptable because the TSAS is quiescent and ubiquitous. This component has been largely neglected in interpretative discussions of the ISM. However, its very existence is antithetical to the standard ISM picture in which the various components have rough pressure equality and, at the very least, pressures low enough to be confined by the Galaxy's gravitational field. Recently, the TSAS has been discussed in terms of very small curved filaments or sheets. These anisotropic geometrical structures reduce the required volume densities and comcomant pressures to acceptable levels. The CNM clouds are permeated by these dense structures and the interstices are occupied by less dense, warmer gas. This is a fundamental change in our concept of the structure of cold interstellar clouds.

The NRAO 140-ft telescope has been used to detect distinct HI clouds towards X-ray shadows, regions where ROSAT results indicate that soft $\mathrm{X}$-rays appear to be absorbed by intervening interstellar matter. Results in the Eridanus region indicate that most of the absorption occurs at a distance of about $100 \mathrm{pc}$, which is consistent with the model in which most of the soft X-ray background originates in a hot bubble of gas surrounding the Sun.

The NRAO 140-ft telescope has been used in a sensitive search for high-velocity HI clouds near and along line-of-sight to QSOs. About $37 \%$ of the observed directions contained high-velocity gas at velocities greater than $100 \mathrm{~km} \mathrm{~s}^{-1}$.

In NRAO 12-m telescope observations probing the ISM, CO J=1-0 absorption has been detected towards some compact extragalactic mm-wave continuum sources. Similar observations have been made with the IRAM interferometer for $\mathrm{HCO}^{+} \mathrm{J}=1-0$ absorption; the absorption was found to be surprisingly common, occurring in $30 \%$ as often as $\mathrm{HI}$ absorption for the same directions.

Molecular Clouds and Star Forming Regions. Large-scale mapping of the molecular clouds in our Galaxy continues. The kinematics of molecular clouds have enabled the determination of bar models from the structure of orbits. SiO masers are good probes of galactic structure. NRO 45-m telescope observations have yielded a $65 \%$ detection rate of $\mathrm{SiO}$ masers in a colour-selected sample of IRAS sources and have been used for an investigation of the kinematics of the galactic bulge. In 1994, FCRAO inititiated a CO J=1-0 survey, using its 14-m telescope to cover $336 \mathrm{deg}^{2}$ of the outer Galaxy at galactic longitudes between $103^{\circ}$ and $145^{\circ}$ and latitudes of $-3^{\circ}$ to $+5^{\circ}$, with sampling interval of 50 arcsec. The Tokyo-NRO $60-\mathrm{cm}$ telescope has been used for a CO J=2-1 survey of the Scutum Arm region of the Milky Way. Thirty four molecular clouds were identified; comparison of $\mathrm{J}=2-1$ and $\mathrm{J}=1-0$ intensities suggested that the spiral arm emission is not dominated by star-forming regions. A survey of CS $\mathrm{J}=2-1$ and its isotopomers suggests that the ${ }^{32} \mathrm{~S} /{ }^{34} \mathrm{~S}$ abundance ratio increases with galactocentric distance.

Several galactic plane surveys of molecular masers have been undertaken. For $\mathrm{CH}_{3} \mathrm{OH}$ masers, Australian telescopes have been particularly active in searches and monitoring of transitions at frequencies of 6.6, 12.2 or $44 \mathrm{GHz}$. A strong anti-correlation between Class I and Class II masers has been noted. A comparison of the 6.6- and $12.2-\mathrm{GHz} \mathrm{CH}_{3} \mathrm{OH}$ masers indicates that individual spectral features of the two transitions often coincide, although with a wide range of relative intensities. The maser intensities are often quite variable, the variability appearing to be quasi-periodic on timescales of between a month and several years. Different features in a maser spectrum usually vary independently. The ATCA has also been used for $\mathrm{OH}$ observations at 1.6 and $6.0 \mathrm{GHz}$. For regions associated with maser emission in different transitions of a molecule or from combinations of $\mathrm{CH}_{3} \mathrm{OH}, \mathrm{OH}$ and $\mathrm{H}_{2} \mathrm{O}$, on a scale of around 1 arcsec the different masers are usually found grouped together at similar positions. It has been suggested that $\mathrm{CH}_{3} \mathrm{OH}$ and $\mathrm{OH}$ are injected into the gas phase by evaporation of grain mantles, and that because photodissociation of $\mathrm{H}_{2} \mathrm{O}, \mathrm{CH}_{3} \mathrm{OH}$ and $\mathrm{OH}$ occurs at similar rates, substantial abundances of $\mathrm{CH}_{3} \mathrm{OH}$ and $\mathrm{OH}$ can co-exist. SEST detection of CS $\mathrm{J}=2-1$ emission towards a sample of masers supports an association with dense molecular cores. Maser emission in the $36-\mathrm{GHz} \mathrm{CH}_{3} \mathrm{OH}$ transition has been detected in several galactic sources with the Pushchino 22-m telescope. The absence of this maser emission in several regions containing 44-GHz masers was explained in terms of high gas density $\left(>10^{6} \mathrm{~cm}^{-3}\right)$. Other $\mathrm{CH}_{3} \mathrm{OH}$ maser transitions have been discovered: Class II masers were discovered at $107 \mathrm{GHz}$ with the OSO 20-m antenna, and a series near $157 \mathrm{GHz}$ with the NRAO 12-m telescope. The detections support an assumption that the population inversion of the energy levels is caused by overpopulation of some $\mathrm{K}$ ladders relative to neighbouring ladders.

JCMT observations have shown that a high-frequency $(662 \mathrm{GHz})$ recombination line emitted by MWC349 is masing; this extends the frequency range in which this phenomenon has been found to occur. 
Many studies have been made of individual star-forming regions, with particular focus on molecular outflows, protostellar condensations, and large-scale distribution. As an example, the NRO 45-m telescope has been used for studies of molecular outflow objects which include IC5146, Haro4-255 FIR, L1251, IRAS $22134+5834, \mathrm{~L} 1287$, and the Taurus complex. It has been suggested that an outflow is a common phenomenon in the formation of stars, including low-mass stars. The detection of $\mathrm{CH}_{3} \mathrm{OH}$ and $\mathrm{SiO}$ in L1157 adds to the number of outflows known to show strong abundance enhancements of these molecules due to evaporation of grain mantles or destruction of dust by shocks.

The NRO 45-m telescope has also been used to study dense cores in Taurus. The detection of many $\mathrm{H}^{13} \mathrm{CO}$ cores led to the suggestion that cores without IRAS counterparts represent protostellar condensations just prior to star formation. A survey of $\mathrm{C}^{18} \mathrm{O}$ emission towards FIR compact sources has revealed a correlation between FIR colour and gas column density. The large-scale ${ }^{13} \mathrm{CO}$ distribution reveals a highly filamentary distribution of star-forming gas.

A multi-transition $\mathrm{H}_{2} \mathrm{CO}$ survey of sub-mm continuum sources in the Serpens cloud shows strong self-reversal features that may indicate infall, and very broad line wings consistent with molecular flows.

Observations of the internal structure of dark cloud cores and globules have begun to clarify the process of low-mass star formation. Observations made at the NASA Complex in Goldstone (USA) of the 22-GHz transition of CCS (and some other molecules) of the dense core TMC1-D have shown it to be composed of many sub-solar-mass clumps. CCS is ideal for this type of study because it exists for only a short period of time as complex molecules begin to form in the core. Thus it highlights young gas regions. It appears that if a solar-mass star is to form at TMC1-D, it will have to be assembled from many smaller clumps. Goldstone, VLA and OVRO observations provide evidence supporting a similar formation in L1498 and B335. In L1498, it appears that an older cloud $\left(\mathrm{NH}_{3}\right)$ is concentrated towards the core and accreting matter from a surrounding young gas containing CCS; CS is concentrated in the interface region. For B335, there is evidence for collapse of the outer cloud containing CCS towards the circumference of a previously discovered central disk.

Other studies are being made of dark clouds such as TMC-1 and L134N. For instance, an intensive study at FCRAO has so far involved more than 30 different molecular transitions of some 20 different molecular species and isotopemers. The aim is to derive information for use in abundance estimates using full statistical equilibrium, LVG analyses. Similar studies of star formation regions in Ori-A, M17 and Cep-A have also been implemented with the FCRAO 14-m telescope.

New molecules $\mathrm{HC}_{3} \mathrm{NH}^{+}, \mathrm{H}_{2} \mathrm{COH}^{+}$and $\mathrm{H}_{2} \mathrm{CN}$ have been detected in TMC-1 with the NRO 45-m telescope. $\mathrm{CH}_{2}$ has been detected in the Orion and W51 interstellar clouds with the NRAO 12-m telescope; it is a parent to several widespread molecules, including $\mathrm{CH}, \mathrm{H}_{2} \mathrm{CO}$, and $\mathrm{HCOOH}$. In addition, $\mathrm{N}_{2} \mathrm{O}$ has been detected towards $\mathrm{Sgr}$ B2; it is one of the few detected molecules containing the $\mathrm{N}-\mathrm{O}$ bond.

NRO $45-\mathrm{m}$ telescope observations of CS $\mathrm{J}=1-0$ in the giant molecular cloud Ori-A produced about 200 cloud cores with generally larger internal turbulent velocities and higher masses compared with the TMC, where low-mass stars are forming. By estimating the mass of the star which would form in each core, an initial mass function (IMF) of stars was derived which was similar to the IMF of field stars for masses greater than 4 times the solar mass. In the molecular cloud near the Orion nebula, densities of molecules such as $\mathrm{CO}, \mathrm{CS}$ and $\mathrm{HCO}^{+}$were found to increase towards the ionization front, and then decrease sharply. The gradual increase was ascribed to compression by shock waves associated with the expansion of the HII region, and the decrease to photo-dissociation of molecules by uv radiation from the stars.

It is believed to be very difficult to form observed abundances of saturated molecules (e.g. $\mathrm{H}_{2} \mathrm{O}$, alcohols) only by ion-molecule reactions, and an NRO 45-m telescope survey of a sample of such molecules in high-mass star-forming regions has indicated that saturated molecules are formed on grain surfaces, and they evaporate from the surfaces by absorbing energy released by newly born stars.

MERLIN polarization studies of $18-\mathrm{cm} \mathrm{OH}$ masers in star-forming regions have revealed ordered magnetic field structures within $1000 \mathrm{AU}$ of the young stars. $\mathrm{OH}$ masers in regions of bipolar outflow show linear polarization in the direction of the outflow, and may also show a magnetic field reversal across the outflow in the direction orthogonal to the bipolar lobes.

NRAO 140-ft observations of $\mathrm{OH}$ towards a sample of compact extragalactic continuum sources revealed $\mathrm{OH}$ emission features with no counterpart in $\mathrm{OH}$ absorption or in spectra of abundant molecules. However, the $\mathrm{OH}$ emission matches deep HI absorption. The results imply that $\mathrm{OH}$ must exist in clouds that are mainly atomic. 
A BIMA image at 0.5 arcsec resolution of the $\mathrm{v}=0, \mathrm{~J}=2-1 \mathrm{SiO}$ line towards Orion-IRC2 shows a bow-tie structure which delineates a 1000-AU diameter flared circumstellar disk. Dense continuum condensations with sizes 500-1500 AU are not common in the Orion molecular ridge or in other similar regions.

Theoretical models predict large abundances of $\mathrm{CO}^{+}$ions at the edges of photon-dominated regions where molecular clouds are exposed to intense stellar far-uv radiation. IRAM 30-m antenna observations of a transition at $236 \mathrm{GHz}$ has confirmed a build-up of $\mathrm{CO}^{+}$emission in the molecular cloud/HII region interfaces in the Orion Bar and M17SW.

The NRAO 140-ft telescope has been used at $8.7 \mathrm{GHz}$ for new measurements of the ${ }^{3} \mathrm{He}^{+}$hyperfine transition in several galactic HII regions. The derived abundances in the range $1-4 \times 10^{-5}$ are not consistent with the overall chemical evolution of the Galaxy, unless local enrichment of some HII regions (e.g. by Wolf-Rayet star winds) has occurred.

SEST observations have revealed the first case of $\mathrm{CO}$ absorption against the CMB - in a bipolar outflow with high mass loss.

IRAM and VLA instruments have been used to study the photodissociation region of the reflection nebula NGC 7023. Interferometric $\mathrm{HCO}^{+}$results delineate four high-density filaments at the interface between atomic and molecular gas; comparison with $\mathrm{HI}$ and single-dish $\mathrm{HCO}^{+}$emission indicates that two of the filaments are on the inner walls of the HI clump, immersed in an atomic medium; the other two are immersed in the molecular cloud.

The dust emission at a wavelength of $1.2 \mathrm{~mm}$ associated with cloud cores has been mapped with several telescopes including the SEST and IRAM 30-m telescope.

Galactic Centre Region. As expected, there has been continuing interest in the central regions of our Galaxy. The central region has been mapped in $\mathrm{CO} \mathrm{J=2-1}$ using the Tokyo-NRO $60-\mathrm{cm}$ telescope. The kinematics indicated that star formation has been occurring, continuously or intermittently, in a region about $100 \mathrm{pc}$ in radius.

The NRO 45-m telescope has been used for several studies of the centre region. CS J=1-0 mapping of the central 150 arcmin $\times 30$ arcmin region revealed several curved filaments extending along the galactic plane, and many expanding shell-like structures (probably due to past star-burst activity). A rotating molecular ring of radius $120 \mathrm{pc}$ has been identified in the longitude-velocity relationship for ${ }^{13} \mathrm{CO} \mathrm{J}=1-0$ emission; a dumbbell-shaped shell was found during the analysis of the three-dimensional structure of this ring.

A 1.6-GHz OH absorption-line survey of the Galactic Centre using the 250-ft Jodrell Bank telescope indicates that the associated molecular gas is more extensive than previously thought - the molecular disk could be traced over the galactic longitude range $354^{\circ}-6^{\circ}$.

Upper limits for the diameter of the Galactic Centre source Sgr $\mathrm{A}^{*}$, believed to be at the dynamical centre of the Galaxy, are 0.4 mas from VLBA observations at $43 \mathrm{GHz}$, and 0.1 mas from VLBI observations at $86 \mathrm{GHz}$. Added to results at other frequencies, JCMT images at $0.8,0.6$ and $0.45 \mathrm{~mm}$ yield a highfrequency spectrum in which flux density varies as frequency ${ }^{1 / 3}$, peaking at a frequency greater that $600 \mathrm{GHz}$ and decreasing rapidly above about $1000 \mathrm{GHz}$. Optically thin emission of quasi-monoenergetic relativistic electrons can account for the spectrum above $1.5 \mathrm{GHz}$, while free-free absorption due to the HII region SgrA West and synchrotron self-absorption in Sgr $\mathrm{A}^{*}$ could account for a spectrum turnover at lower frequencies. The structure around $\mathrm{Sgr} \mathrm{A}^{*}$ includes a central cavity within a radius of $1 \mathrm{pc}$, a circum-nuclear disk out to $12 \mathrm{pc}$, as well as the well-established minispiral with bar.

VLA $\mathrm{H}$ recombination-line observations at 6 and $8 \mathrm{GHz}$ have been used to investigate the kinematics of the ionized gas within the inner few parsecs. New kinematic components were identified with velocities quite different from those consistent with circular motion about the dynamical centre - an ionized counterpart to a highly blueshifted molecular cloud, large scale linear ionized 'streamers'. One study targeted the inner parsec, in particular near the 'minicavity' which appears as a hole in the distribution of the streamers.

New observations have been made of the molecular clouds towards $\mathrm{Sgr}$ A. 5- $\mathrm{GHz} \mathrm{H}_{2} \mathrm{CO}$ observations with the VLA indicate that the molecular clouds are gravitationally stable at present, but will have lifetimes smaller than $10^{6}$ yr because of the tidal forces from Sgr A*. CS J=1-0 emission at $50 \mathrm{~km} \mathrm{~s}^{-1}$ has been mapped with the NMA. The CS emission was found to be resolved into a few kinky and curved filaments embedded in an expanding shell-like structure, suggesting interaction of the cloud with an expanding HII region. $\mathrm{HI}$ and $\mathrm{OH}$ observations of the $-180 \mathrm{~km} \mathrm{~s}^{-1}$ molecular cloud suggest an origin as a 
tidally disrupted high-velocity cloud flowing towards the Galactic Centre. Several $1720-\mathrm{MHz}$ OH masers, usually found around the outer edges of SNRs, have been recently detected in the outer regions of the Sgr A complex. VLA searches in the inner few arcmin of the Galactic Centre region has detected five $\mathrm{H}_{2} \mathrm{O}$ masers. Three are associated with evolved stars, one is located in the Sgr A East HII region, and the fifth is 30 arcsec north, and 35 arcsec east, of $\mathrm{Sgr} \mathrm{A*}$, near the eastern end of the radio minispiral. The last maser coincides with a luminous reddened star believed to be an $M$ supergiant; its velocity is similar to that expected for gas in the circumnuclear disk at this location.

The HII emission and dense molecular clouds of Sgr B2 continue to be popular targets for research. Sensitive observations have been made towards the continuum emission at $3 \mathrm{~mm}$ and $7 \mathrm{~mm}$. VLA imaging of continuum and recombination-line $(\mathrm{H}$ and $\mathrm{He})$ at $1.3 \mathrm{~cm}$ has provided new information on the circumstellar environments in the main complexes. A SEST spectral-line survey of line absorption against the main continuum region Sgr B2(M) in the frequency range $81-113 \mathrm{GHz}$ yielded detections of 17 transitions. Spectral-line observations have been made for several molecules and their isotopomers, including $\mathrm{CH}_{3} \mathrm{COOH}, \mathrm{CCS}, \mathrm{CH}_{3} \mathrm{COCH}_{3}, \mathrm{CS}, \mathrm{HC}^{13} \mathrm{CCN}, \mathrm{HC} 3 \mathrm{OH}, \mathrm{HCl}, \mathrm{H}_{2} \mathrm{CO}, \mathrm{NH}_{3}$ and $\mathrm{SiO}$. The 625- $\mathrm{GHz} \mathrm{HCl}$ transition was observed from the NASA Kuiper Airborne Observatory. The $\mathrm{NH}_{3}$ observations include a multilevel study and investigation of a hot shocked $\mathrm{NH}_{3}$ region. Similarities between the SiO absorption and the absorption in the $(8,8)$ and $(9,9)$ transitions of $\mathrm{NH}_{3}$ observed with the Effelsberg 100-m telescope suggest that the $\mathrm{SiO}$ absorption arises in the same extended hot $(175 \mathrm{~K})$ low-density envelope as the $\mathrm{NH}_{3}$. In some cases the molecular distributions were mapped over the entire Sgr B2 complex. ${ }^{13} \mathrm{CO} \mathrm{J=1-0}$ mapping with the NRO $45-\mathrm{m}$ telescope has provided evidence to support a cloud-cloud collision, and consequential triggering of star formation. New $6.7-\mathrm{GHz} \mathrm{CH}_{3} \mathrm{OH}$ and 4.8-GHz $\mathrm{H}_{2} \mathrm{CO}$ masers have been detected, some in positions containing other masers or compact continuum components. In high-resolution CS $\mathrm{J}=2-1$ imaging of Sgr B2(M), NMA observations were interpreted in terms of rotation, whereas BIMA results have been discussed in terms of a high-velocity molecular outflow. VLA continuum and $\mathrm{H}$ recombination-line observations of the numerous components in this region do not support HII region formation by moving-star bow shocks. BIMA observations have revealed a hot young molecular core of diameter below $0.1 \mathrm{pc}$ (i.e. below $2.5 \mathrm{arcsec}$ ) in another core $\mathrm{Sgr} \mathrm{B} 2(\mathrm{~N})$. The detection of spectral lines of interstellar $\mathrm{CH}_{3} \mathrm{COCH}_{3}$ and $\mathrm{CH}_{3} \mathrm{COOH}$ were towards this core; the presence of such complex molecules highlights the importance of grain chemistry here. VLA observations of the HI Zeeman effect towards Sgr B2(M) and Sgr B2(N) have yielded a line-of-sight magnetic field strength of $-0.5 \mathrm{mG}$, with spatial variations of $50 \%$.

The BIMA array had been used to produce a 6 -field mosaic image of $98-\mathrm{GHz}$ CS emission toward the HII region G0.18-0.04 (the 'Sickle'). A multifield MEM deconvolution of the 3 arcmin $\times 4$ arcmin field recovered much of the large-scale structure often missed in linear mosaic techniques. Several streamer-like structures associated with non-thermal filaments were revealed, where gas is piling up as the cloud is braked by interaction with the magnetic field.

A nonthermal filament (G359.1-0.2, the 'Snake') was discovered from the MOST survey of the Galactic Centre region. This object has several narrow strands, has high linear polarization over part of its length (which is kinked), and is considered to contain strong magnetic fields parallel to its extension. Its origin is still unclear.

\subsection{EXTRAGALACTIC PHENOMENA}

\subsubsection{Large and Small Magellanic Clouds}

Continuum Observations. Being the nearest supernova to have been detected in the last 400 years, SN 1987A in the LMC has provided a unique opportunity to study not only a supernova event and its aftermath, but also the progenitor star and its circumstellar medium. MOST observations at 843 $\mathrm{MHz}$ have provided the most comprehensive time sampling of the radio emission, and the history of the observations up to September 1994 has been reported. A brief radio outburst was detected a few days after the explosion, but subsequent redetection, by both the MOST and ATCA, did not occur until mid 1990. The flux density has steadily increased, and is now above $70 \mathrm{mJy}$ at $1.4 \mathrm{GHz}$; however, the spectral index remains constant at $\mathbf{- 0 . 9}$. The sudden increase of radio emission several years after the explosion could be due to the supernova shock encountering a sudden increase in the density of circumstellar material associated with past mass-loss history of the progenitor. A 9-GHz ATCA image of the remnant, produced by super-resolution with an effective resolution of 0.5 arcsec, shows shell-like emission with two bright lobes on opposite sides of the shell. Optical observations suggest that the lobes correspond to the 
equatorial plane of the progenitor. The remnant is expanding at about $3,000 \mathrm{~km} \mathrm{~s}^{-1}$, much more slowly than the initial shock velocity of around $30,000 \mathrm{~km} \mathrm{~s}^{-1}$. A model of the radio emission has been based on diffusive shock acceleration of electrons and ions by the supernova blast wave.

The Parkes 64- $\mathrm{m}$ telescope has been used in a survey of radio sources towards the LMC at frequencies of $1.40,2.45,4.75$ and $8.55 \mathrm{GHz}$. Together with information obtained from an analysis of the $4.85-\mathrm{GHz}$ Parkes-MIT-NRAO survey, $\mathbf{4 6 9}$ objects were detected. Images and source lists from a MOST survey of the SMC are now available.

Several SNRs in the LMC and SMC have been imaged with the ATCA. One of the LMC objects, DEM L316, shows two overlapping shells. Enhanced O III emission at the juncture of the shells, enhanced $\mathrm{X}$-ray emission from one shell at the juncture, and a change in the structure of the magnetic field near the interacting region, together strongly support a collision between the two remnants.

HI Observations. The ATCA has been used in mosaic mode to survey the HI in the SMC over an area of 20 square degrees with an angular resolution of 1.6 arcmin and rms brightness sensitivity of $1.4 \mathrm{~K}$ (corresponding to an HI column-density sensitivity of $4 \times 10^{18} \mathrm{~cm}^{-2}$ for each velocity channel of width $1.6 \mathrm{~km} \mathrm{~s}^{-1}$ ). The HI distribution was found to be complex and, on scales $\sim<1 \mathrm{kpc}$, dominated by the effects of expanding $\mathrm{HI}$ shells which are probably driven by the combined effects of supernovae and stellar winds from massive stars. The results seem to challenge the earlier belief that the SMC consists of two or more spatially separate structures with different systemic velocities - in many cases the observed multiple components are caused by the combined effects of the numerous shells and supershells. Six supershells (radii greater than $300 \mathrm{pc}$ ) and 495 giant shells were identified. The apparent age distribution of shells has a mean age of $5.4 \mathrm{Myr}$ and an intrinsic dispersion of 2.6 Myr. Southern shells appear to be older, on average, by $2.5 \mathrm{Myr}$. The kinetic energy of the shells is a large fraction of the gravitational binding energy of the SMC, implying that further disintegration of the SMC will occur with time unless the SMC possesses a massive halo.

The ATCA was also used for an HI survey of the LMC; a field of $10^{\circ} \times 12^{\circ}$ was imaged with an angular resolution of $60 \operatorname{arcsec}(\sim 15 \mathrm{pc})$ and a velocity resolution of $1.6 \mathrm{~km} \mathrm{~s}^{-1}$. The images show the detailed spatial structure of the neutral interstellar gas for the first time. On small to medium scales, the combined action of numerous shells and supershells dominate the structures and motions. On large scales, the galaxy is remarkably symmetrical in appearance compared with other wavelengths and shows a pronounced spiral pattern. A southern spiral arm is seen for the first time.

The ATCA has also been used for a study of the cool atomic gas in the LMC based on observations of $\mathrm{HI}$ absorption.

Molecular Cloud Observations. Several research groups are using the SEST at wavelengths of 3, 2 and $1 \mathrm{~mm}$ for molecular cloud searches. The installation of a new, more sensitive 3 - and 2-mm receiving systems has resulted in the detection of around 20 molecular transitions; the detections include the first extra-galactic $\mathrm{SiO}$ maser - in the $\mathrm{OH} / \mathrm{IR}$ object IRAS 04553-6825, and the first definite discoveries of extragalactic deuterium ( $\mathrm{DCO}^{+}$and $\mathrm{DCN}$ ) in LMC star-forming regions $\mathrm{N} 44, \mathrm{~N} 113$ and $\mathrm{N} 159$. A study of $\mathrm{CO} \mathrm{J=1-0}$ and IRAS maps of LMC molecular clouds has indicated that although less luminous than their counterparts in our Galaxy, the clouds are nevertheless supporting massive star formation. In an ATCA survey of regions selected on the basis of IRAS colours, a third $6.6-\mathrm{GHz}^{\mathrm{C}} \mathrm{CH}_{3} \mathrm{OH}$ maser was detected, towards IRAS 05011-6815. In addition, the first detected extragalactic LMC 6.0-GHz OH maser was observed near the HII region $\mathrm{N} 160 \mathrm{~A}$.

\subsubsection{Other Relatively Nearby Galaxies.}

Continuum Emission and Structure The Effelsberg 100-m telescope is an extremely sensitive instrument for detecting extended continuum emission and molecular line emission from galaxies. It has been used for 2.8-cm observations of all Shapley-Ames galaxies north of declination $-25^{\circ}$. The results have provided statistics on the relationship of the correlation between radio continuum and far-infrared (FIR) emission to the average magnetic strengths of normal galaxies. The latter do not appear to correlate with the average synchrotron spectral indices, suggesting that the galaxies lose their cosmic-ray electrons mainly via escape. It has been concluded that a close coupling between gas clouds and magnetic fields, plus a star-formation rate related to gas density, can explain the radio-FIR correlation. 
As part of the VLA compilation of a $1.425 \mathrm{GHz}$ atlas of the IRAS bright galaxy sample, galaxies north of declination $-45^{\circ}$ and more than $10^{\circ}$ off the galactic plane have been observed.

Observations have been made with the VLA at 3.6 and $6 \mathrm{~cm}$ and with MERLIN at $18 \mathrm{~cm}$ of a sample of mildly active galaxies including the archetypal starburst galaxies NGC 253 and 1808 . The spectral indices of the prominent compact components near the nucleus are consistent with the components being SNRs.

A sample of galaxies in the Virgo cluster was found to show excess radio emission due to interaction.

M 31 has been given special attention. Observations with the VLA and Effelsberg 100-m telescope show a spectral index between 20 and $6 \mathrm{~cm}$ decreasing from -0.1 in the centre to -0.7 at a galactocentric distance of $1.5 \mathrm{arcmin}$; this is similar to the nuclear regions of other galaxies. A tight correlation has been found to exist between 20-cm continuum emission and total FIR brightness, even on a scale of $1 \mathrm{kpc}-$ the thermal component correlates with the warm FIR, and the non-thermal component with the cool FIR.

6-cm MERLIN observations, with a resolution of 50 mas, have yielded images of $\sim 25$ SNRs in M 82 . Many show clear shell structures and the majority are both more luminous and more compact than CAS A, suggesting ages of order 100 years. Cumulative diameter-number plots give estimates of supernova rates of 0.05 per year. Recent $20-\mathrm{cm}$ and $73-\mathrm{cm}$ MERLIN observations show many of the remnants to have low-frequency turnovers consistent with free-free absorption by ionized gas. HI-absorption measurements have been made against the stronger remnants.

In MOST studies of spiral galaxies, a sample is being searched for radio supernova using existing early observations and a second-epoch set. The relationship between radio cores and the radio emission from outer regions is being investigated.

Magnetic Fields. Most of our knowledge of magnetic fields in galaxies is derived from observations of the linear polarization of synchrotron radiation at $\mathrm{cm}$ wavelengths. The observed direction of polarization suffers Faraday rotation due to the magneto-ionic material along the line-of-sight, but the intrinsic direction, which is orthogonal to the magnetic field direction, can be determined from observations at two or more wavelengths. Magnetic field strengths are generally calculated assuming equipartition between the energy densities of cosmic ray particles and magnetic fields.

The Effelsberg telescope is effective for detecting weak polarized emission and thus regular magnetic fields from galaxies. Polarization observations of a sample of edge-on galaxies (including NGC 253) have yielded magnetic fields mainly parallel to the galaxy plane, in agreement with dynamo theory predictions. M 82, NGC4631 and the Circinus galaxy are rare exceptions where radial magnetic fields dominate, possibly indicating a dynamo field modified by a strong galactic wind. The degree of polarization in halos is much lower than expected if the correlation length of the random field is similar to that in the disks; however, it appears that correlation length increases with distance off the plane.

The magnetic field structure and its relationship to spiral arms has been investigated in several face-on galaxies, including M 83 with the Effelsberg 100-m telescope and ATCA, IC 342 and NGC 2276 with the VLA, and NGC 1566, and NGC 1672 with the ATCA. It has been concluded that the magnetic fields do not show simple geometrical symmetry because they are strongly affected by spiral arms, bars and gravitational interactions. Effelsberg and VLA observations of NGC 6946 have revealed highly aligned magnetic fields concentrated in two main spiral features between the optical spiral arms. Such magnetic arms are in conflict with the predictions of current dynamo and density-wave models. Observations of $M$ 51 with these telescopes at four frequencies have delineated a magneto-ionic halo, of radial extent about $10 \mathrm{kpc}$, in which the field is mainly parallel to the plane with an inward spiral direction. In contrast, the disk field can be described by superposition of an axisymmetric and bisymmetric dynamo mode.

In a 20-cm VLA survey of M 31, 36 linearly polarized background sources were detected which will serve as probes for studies of the ionized gas and magnetic fields of the halo of the galaxy.

A close relationship between radio continuum emission (Effelsberg 100-m and VLA observations) and $\mathrm{CO}$ emission (IRAM 30-m observations) has been interpreted in terms a coupling of magnetic fields to gas clouds.

A short review of magnetic fields in spiral galaxies is included in the report of Commission 28.

HI, Molecules and Dust. HI studies of galaxies using several telescopes have continued, with projects involving samples of barred galaxies, elliptical galaxies, extreme late-type galaxies, galaxies in groups 
(e.g. Coma I cloud, Fornax cluster), interacting galaxies, IRAS galaxies, lenticular galaxies, polar-ring galaxies, low-luminosity galaxies, nearby galaxies, Seyfert galaxies, and spiral galaxies.

$\mathrm{HI}$ has been detected with the Effelsberg $100-\mathrm{m}$ telescope in a number of elliptical galaxies; no new HIrich dwarf galaxies were found in the group. An extended $\mathrm{HI}$ halo was detected around the irregular galaxy NGC 4449. Galaxies studied with the VLA include NGC 1808. The ATCA has been used for observations of several southern galaxies and groups of interacting galaxies; the targets include the prominent spiral galaxies NGC 253, NGC 1313, NGC 1566, NGC 1672, NGC 2442, NGC 4945, Circinus galaxy. An ATCA study is underway of HI absorption towards galactic nuclei containing radio continuum emission.,

Several groups have searched for extragalactic HI from galaxies optically obscured by the galactic plane. Several nearby galaxies have been detected, two with the Dwingeloo 25-m telescope (the Sagittarius dwarf galaxy, Dwingeloo 1), one with the WRST (Dwingeloo 2), and four with the Effelsberg 100-m telescope (Cas-1, Cas-2, MB1 and Cam-B, all possible members of the IC 342/Maffei group of galaxies).

HI observations with the VLA towards several galactic systems believed to be in progressive stages of merging (Arp 295, NGC 4676, NGC 520, NGC 3921 and NGC 7275) have suggested several merging trends. In the beginning, large amounts of HI still exist within the galactic disks and star formation is widespread; ionized gas emission often appears as plumes and arcs emanating from the nuclear regions. In the final stages, little or no HI is in the remnant bodies, and tidal material is seen moving inward.

Observations of molecular gas (in particular $\mathrm{CO}$ ) play an essential role in the study of the origin, evolution, structure and kinematics of galaxies. $\mathrm{CO}$ observations of galaxies have been obtained with several radio telescopes. The IRAM 30-m and NRO 45-m telescopes have been used to survey the CO emission in a number of nearby spiral galaxies; special attention has been given to $M$ 51, the results suggesting that star formation is triggered by molecular cloud collisions in the spiral arms but by selfgravity of clouds in the inter-arm regions. Several southern spiral galaxies have been studied with the SEST. Rotating CO rings appear to be present in the nuclei of galaxies NGC1808, NGC 4414, NGC 4565, NGC 4631 and NGC 4945. Imaging of ${ }^{13} \mathrm{CO}$ in M 82 with the IRAM interferometer has shown, in addition to a molecular-ring sub-structure, velocity-field distortions attributable to an expanding shell centred on a compact continuum source considered to be a supernova remnant.

Because of its proximity, M 31 should be an ideal galaxy for a study of molecular clouds and their relationship to galactic structure. However, this has been hindered by the weakness of $\mathrm{CO} \mathrm{J}=1-0$ emission in the galaxy. Nevertheless, such studies are continuing. For instance, an on-going CO study of $\mathrm{M} 31$ with the IRAM 30-m telescope shows evidence of low-temperature molecular clouds near the giant star cluster NGC 206 and a patchy distribution of gas in spiral arms. A major CO study is underway with the FCRAO 14-m telescope and its QUARRY multi-beam array. To date, the $\mathrm{CO}$ distribution has been mapped along a major portion of a spiral arm; a 200-pc offset was found between the gaseous spiral arm and the HII regions.

A compilation of rotation curves of nearby spiral galaxies based on $\mathrm{CO}, \mathrm{HI}$ and optical results shows that the inner rotation curves are generally characterized by a steep gradient within a radius of a few hundred parsecs, indicating a compact massive concentration near the nucleus. Evidence has been presented for the existence of a molecular front, a critical radius of a few $\mathrm{kpc}$ within which the galaxy disk is almost totally molecular, and beyond which the interstellar gas is almost all HI. It has been suggested that the CO-to- $\mathrm{H}_{2}$ conversion factor is related to the metallicity of molecular clouds in a galaxy.

It has been suggested that, since atomic and molecular gas share the same galactic rotation, a TullyFisher Relation using $\mathrm{CO}$ instead of $\mathrm{HI}$ should be possible, and might be even more suitable for cosmological distances, since $\mathrm{CO}$ can be detected in more distant galaxies than $\mathrm{HI}$. Recent $\mathrm{CO}$ results appear to support this.

Dust continuum has been observed in a number of elliptical and spiral galaxies, and some mapping has been carried out. It has been detected from the disks of edge-on galaxies NGC 891 and NGC 4631, from the spiral arms of M 51, and also from a few protogalaxy candidates. IRAM 30-m antenna observations of edge-on galaxy NGC 4565 reveal $1.2-\mathrm{mm}$ continuum emission (from dust) that follows CO near the nucleus and $\mathrm{HI}$ at the periphery; the results are interpreted as the first unambiguous detection of dust emission in extragalactic HI clouds. An estimated value for the $\mathrm{H}_{2} / \mathrm{CO}$ conversion factor was about four times smaller than for the generally accepted galactic value, but similar to values derived for other galaxies.

Other comparative studies of dust emission (observations at $1.3 \mathrm{~mm}$ ) and $\mathrm{CO}$ have been made using the IRAM $30-\mathrm{m}$ telescope and SEST for flux-limited groups of active and non-active galaxies. It was 
found that the CO luminosities and 1.3-mm fluxes correlate, that the emission is concentrated towards the nucleus of starburst galaxies but extended along the spiral disk of non-active galaxies, and that the ratio of total IR luminosity to gas mass is about twenty times higher for active galaxies.

CO J=1-0 observations with the IRAM interferometer of NGC 1530 show abundant gas associated with the dust lanes along the galaxy's bar. Near the nucleus are found strong shock fronts and a nuclear ring, but no gas at the nucleus; the shock fronts are barely resolved perpendicular to the bar. The gas appears to follow elliptical orbits along the bar, with strong deviations at the shock fronts and near the nucleus.

Transitions of several molecules, at wavelengths mainly near $3 \mathrm{~mm}, 2 \mathrm{~mm}$ and $1 \mathrm{~mm}$, have been detected with single-dish telescopes in a small number of galaxies, including NGC 253, NGC 4945 and M 82. For some molecules, more than one transition has been observed. OCS has been detected in the centre of NGC 253; this is the heaviest molecule detected in external galaxies so far. Two galaxies have shown evidence of $\mathrm{HCO}$, a possible new extragalactic molecule.

Millimetre-wave interferometers are being used for high angular resolution studies of the molecular content of galaxies, particularly in the nuclear regions. BIMA has been used for imaging of 3-mm molecular lines, for example CS in NGC 253. CO and HCN observations with the NMA have also been used to probe circumnuclear regions of nearby galaxies. For NGC 106, a molecular bar was detected that is similar to the 2- $\mu \mathrm{m}$ stellar bar; the most intense $\mathrm{CO}$ is located typically 15 arcsec from the nucleus along the inner spiral arms and not in a ring.

The four 1.6-GHz transitions of $\mathrm{OH}$ have been detected with the ATCA in the nuclear region of NGC 5128 (Cen A). Whereas the two 1665- and 1667-MHz mainline transitions are in absorption, the 1612 and $1720-\mathrm{MHz}$ satellite transitions show both emission and absorption, but conjugately for each velocity component.

Recombination-line Observations. After a long gap of about 15 years since the discovery (in 1977) of radio recombination lines from two extragalactic objects (M 82 and NGC 253), new detections were made in several starburst galaxies (NGC 660, NGC 1365, NGC 2146, NGC 3256, NGC 3628, NGC 3690, NGC 4151, NGC 4945, IC 694, M 83, Arp 220, and Circinus galaxy). Most of the detections have been made at $\mathrm{cm}$ wavelengths using the VLA and the ATCA. Recombination-line emission at $\mathrm{mm}$ wavelengths has also been observed for some of these galaxies, using the SEST, IRAM 30-m telescope, and the JCMT. All the observations indicate the presence of high-density $\left(>10^{-3} \mathrm{~cm}^{-3}\right)$ ionized gas in the nuclear starburst regions. A high-resolution recombination-line image of NGC 253 has revealed the existence of orthogonal rotating disks in the nuclear region.

Supernova Remnants. The VLA, VLBA, EVN, and several NASA Deep-Space Network antennas were used to monitor the expanding shell of Supernova 1993J in M 81. A sequence of high-resolution VLBI images obtained over a period of a year show symmetrical expansion, although the radio emission is clearly stronger on one side of the shell.

\subsubsection{Galaxy Clusters, Mergers, Interactions etc}

Radio Halos in Clusters. The radio halos associated with clusters of galaxies are extended diffuse radio sources with no optical counterpart, typical sizes of $1 \mathrm{Mpc}$, and steep radio spectrum. Despite numerous searches, few have been detected. The prototype of this class of object is Coma-C, centred on the Coma cluster. In a few cases, sources with similar properties have been found at cluster peripheries; these are called "relic" sources.

Clusters containing radio halos have high richness but no strong galaxy concentration, high X-ray luminosity, no cooling flow, and high temperature $(7-14 \mathrm{Kev})$. The origin of halos is not fully understood. A merger process is supported by X-ray and optical data, and would disrupt any cooling flow. However, mergers appear to be common whereas radio halos are rare. A study of Coma-C suggests that the particle reacceleration mechanism is more efficient in the inner cluster region containing the tailed radio galaxy NGC 4869. If this galaxy were responsible for the supply of relativistic electrons to the halo, the need for a tailed galaxy at the cluster centre to supply the particles could explain the rarety.

It has been found that the Perseus cluster, which has a strong cooling flow at its centre, contains only a small weak radio halo around the central galaxy. It is suggested that cooling flows can suppress the 
diffusion of turbulently amplified B-fields outward from the cluster core, leading to the development of minihalos confined to the core.

The VLA has been used for polarimetric observations of radio galaxies embedded in extreme cooling flow clusters. In some cases, Rotation Measures higher than $800 \mathrm{rad} \mathrm{m}^{-2}$ were measured; the high values are likely to be produced by magnetic fields associated with the dense, hot $\mathrm{X}$-ray emitting gas in the cooling flow clusters.

HI surveys of more distant galaxies have been undertaken in studies aimed at the structure of the Universe - eg galaxies associated with the Bootes void, the Hercules supercluster etc.

In other studies continuum observations of a region containing the Coma cluster have been made at eight frequencies with the DRAO array and VLA. Only 29 radio sources were identified with Coma galaxies. The MOST has been used for a comprehensive survey of Abell clusters and comparison of results with optical and X-ray data.

CO observations of galaxy clusters have shown that the "cooling flows" are much weaker than assumed from X-ray observations, so that the molecular gas cannot originate from the cooling flow.

Molecular-line surveys of pairs of galaxies and Hickson compact groups have yielded evidence of star-formation triggering, and enhancement of $\mathrm{CO}$ emission through tidal interactions.

\subsubsection{Megamaser Galaxies}

Megamasers have been detected for four molecules: $\mathrm{OH}, \mathrm{CH}, \mathrm{H}_{2} \mathrm{CO}$, and $\mathrm{H}_{2} \mathrm{O}$. In all cases the line emissions are found at the galactic nuclei and depend strongly on the type of activity in the nucleus. After the early $\mathrm{OH}$ megamaser searches among luminous FIR galaxies during the 1980 's, $\mathrm{H}_{2} \mathrm{CO}$ megamasers were found in twelve galaxies with similar FIR/nuclear characteristics as the $\mathrm{OH}$ megamaser galaxies. There is evidence that the megamaser emission results from amplification of background continuum by foreground molecular gas, which is part of a edge-on torus. Recent extragalactic searches for the $\mathrm{CH}$ radical have not resulted in any published results, while extragalactic searches for $\mathrm{CH}_{3} \mathrm{OH}$ maser emission at $6.7 \mathrm{GHz}$ has yielded only standard Class II masers in the nearby LMC similar to those in the Galaxy.

OH Megamasers. VLBI studies of the prototype OH megamaser Arp 220 (IC 4553) shows very compact components in its western nucleus. Because of this result, it has been suggested that the high brightness emission originates in a compact circumnuclear disk and that the observed disk size represents the radial distance of the masering gas. This would be similar to the situation in $\mathrm{H}_{2} \mathrm{O}$ megamasers like NGC 4258 . A VLA study of the $\mathrm{H}_{2} \mathrm{CO}$ emission, combined with earlier VLBI OH observations, indicates offsets of the high brightness maser components from the continuum peaks at both nuclei. The apparent spatial offsets would be consistent with amplification by foreground circumnuclear gas but not support an origin within a compact circumnuclear disk. The velocity field defined by the maser emission provides a first insight into the dynamics of the two merging nuclei of the galaxy.

Higher resolution MERLIN data of $\mathrm{OH}$ megamaser IIIZw35 show considerable structure in its emission region, suggesting that the maser emission is associated with a large fraction of the nuclear region.

OVRO mm-array observations of $\mathrm{CO}$ emission in the megamaser galaxy Mrk 273 show an extended component with a rotational velocity gradient plus unresolved gas complex coincident with the optical nucleus. The results are coincident with the interpretation of the galaxy as a young merger system.

Attempts to detect $\mathrm{OH}$ Zeeman splitting in several megamaser galaxies using the ATCA have been unsuccessful.

Theoretical studies of $\mathrm{OH}$ megamaser activity confirms the FIR pumping schemes proposed in the past. The dominant $1667-\mathrm{MHz} \mathrm{OH}$ lines observed in the megamasers and the observed $1665-/ 1667-\mathrm{MHz}$ flux ratios can be explained by radiative pumping of compact clouds $(3 \mathrm{pc}$ ) with temperatures above 40 $-50 \mathrm{~K}$ and $\mathrm{H}_{2}$ densities between a few times $10^{3} \mathrm{~cm}^{-3}$ and a few times $10^{4} \mathrm{~cm}^{-3}$. Larger clouds covering the nucleus may account for the observed absorption in higher $\mathrm{OH}$ transitions.

$\mathrm{OH}$ megamasers are generally found in rapidly evolving and/or merging galaxy systems in which rapid star-formation processes provide the energy source for the FIR/dust emissions. Modelling of the evolution of these galaxies suggests that $\mathrm{OH}$ megamaser activity occurs in the early stages of the mergers or interactions. A small number of powerful 'gigamasers' with $\mathrm{OH}$ luminosities greater than $10^{4}$ solar luminosities has now been found at increasing redshifts of up to $z=0.265$. Limited surveys suggest that $\mathrm{OH}$ megamaser activity would be rather common among luminous FIR sources at high redshifts. Such objects should be easily detectable with existing high-sensitivity telescopes. The powerful mega- 
or gigamasers that have been observed at low redshifts would be detectable beyond the $\mathrm{z}=3$ barrier, at redshifts where QSOs are born. However, the success of such searches will depend strongly on efforts to clean up the interference environment below $2 \mathrm{GHz}$.

$\mathrm{H}_{2} \mathrm{O}$ Megamasers. A number of new searches have been made for $22-\mathrm{GHz} \mathrm{H}_{2} \mathrm{O}$ megamasers. There are now seventeen galaxies with these megamasers in their nuclei: Mrk 1, NGC 1052, NGC 1068, NGC 1386, Mrk 1210, NGC 2369, NGC 3079, IC 2560, NGC 4258, NGC 4945, NGC 5347, Circinus Galaxy, NGC 5506, ESO 103-G35, IRAS F22265 1826, IC 1481 and M 51. Eleven of these have been discovered during the last three years from surveys of about 300 galaxies. VLBI observations of three have revealed disk structures in Keplerian rotation. The archetypal megamaser galaxy is NGC 4258. The serendipitous discovery with the Nobeyama $45-\mathrm{m}$ telescope of maser features displaced $1000 \mathrm{~km} \mathrm{~s}^{-1}$ in velocity each side of the main features at the systemic velocity, was followed by VLBA/VLA observations which indicated that the high-velocity components conform to a nearly perfect Keplerian system to an rms accuracy of 3 $\mathrm{km} \mathrm{s}^{-1}$ for a mean rotation velocity of $100 \mathrm{~km} \mathrm{~s}^{-1}$. A central mass of 35 million solar masses is required, contained within a disk of radius $0.13 \mathrm{pc}$. The disk is very thin, warped slightly and probably responsible for the dominance of the red-shifted masers. Preliminary measurements of the centripetal accelerations of the maser components and their proper motions give a galaxy distance of about $7 \mathrm{Mpc}$. VLBI observations of NGC 1068 and NGC 4945 suggest similar, but not well-established, structure. It has been suggested that the $\mathrm{H}_{2} \mathrm{O}$ masers in NGC 2369 also originate in a dense nuclear accretion disk: although no highvelocity features have been detected, a velocity drift of maser features has been observed which could be due to the centripetal acceleration of clumps of gas in an accretion disk. The results have stimulated further searches for other systems. New Parkes observations of the Circinus Galaxy have revealed a group of features with velocity about $250 \mathrm{~km} \mathrm{~s}^{-1}$ lower than the main emission discovered about fifteen years earlier, and the two groups have velocities symmetrically offset from the systemic velocity.

\subsubsection{Radio Galaxies, AGNs and Quasars}

Nuclear Structure. The BIMA array has been used for the first survey of Seyfert nuclei at a radio resolution of several arcsec. To date, all observed objects have nuclear $\mathrm{CO}$ concentrations with implied surface densities hundreds of times greater than in the disk of our Galaxy. Strong nuclear asymmetries $(\mathrm{m}=1$ mode) are present in many cases. The prototype Seyfert galaxy NGC 1068 shows a molecular bar and a nuclear concentration of $\mathrm{HCN}$ in contrast to the more extended $\mathrm{CO}$ from the kpc-scale starburst region.

SEST observations of the radio galaxy Cen $\mathrm{A}$ have revealed a nuclear disk of $\mathrm{CO}$ and other molecules with a radius of $200 \mathrm{pc}$.

HI absorption has been detected with the VLA against the central radio source of Cyg A. Together with additional evidence from NGC 4151, this has provided support for a nuclear atomic obscuring torus.

$X$-ray Environment. Significant advances (if not breakthroughs) have been made by X-ray observations of radio galaxies to study the interaction of radio jets, hotspots, and lobes with the ambient gas. Studies of objects such as Cyg A and their hotspots have provided a new measure of average B field, evidence that there is little entrainment, better understanding of bow shocks, and constraints on the jet fluid (protons vs positrons).

The non-thermal energy density in the interior of extended radio galaxies cannot be determined unambiguously on the basis of the radio data alone. A convenient estimate is the minimum value (equipartition value), provided by radio data when the contributions from the relativistic particles and magnetic fields are approximately equal. This value depends on assumptions on the geometry, including the filling factor, and the ratio between the contributions of the electrons and the protons. Supplementary information is needed to verify if the internal energy is really close to its minimum value. Such information can be obtained for the extended and apparently relaxed components of radio galaxies, which are likely to be statically confined by the surrounding medium, particularly when the temperature and density of this medium can be derived from X-ray data.

The comparison between the internal equipartition pressure in radio emitting regions and the external thermal pressure provided by the $\mathrm{X}$-ray emitting gas has been performed for some low-luminosity radio galaxies and tailed radio sources. The internal pressure in the outer radio regions was found to be lower than the ambient pressure. The reason for this apparent imbalance may be due to the assumptions 
associated with the calculation of equipartition parameters, an absence of equipartition, or presence of thermal plasma within the radio lobes.

In addition to the results for hotspots, an independent measure of $B$ fields in radio sources has been provided by inverse Compton $\mathrm{X}$-rays from the $3-\mathrm{K}$ microwave background. A reasonably strong case has been presented, based on ROSAT and ASCA data, for the detection of this inverse Compton component from the lobes of For A.

Jets. A global VLBI/VLBA study of a complete sample of 27 radio galaxies of low or intermediate radio power has been made to derive constraints on jet velocity and orientation with respect to the line-of-sight, with the aim of testing the predictions of the unified scheme model. It was found that low-power (FR I) radio galaxies always show an asymmetric parsec-scale morphology (core and a one-sided jet), with the nuclear emission as the dominant component. This morphology is similar to that for high-power (FR II) radio galaxies. These jets do not show large bends and are always orientated as the main kpc-scale jet. The results are consistent with the expectations of unified models. The observations suggest jets with an intrinsic Lorentz factor equal to or greater than 2, viewed at angles larger than 30 degrees (although in some cases smaller angles with lower jet velocity cannot be excluded). FR I galaxies appear to be the parent population of BL-Lac objects. Three objects fell outside the classification - two (3C338 and $1144+35$ ) with two-sided parsec jets or lobes. The object 4C31.04 appears to be a low-power member of the class of Compact Symmetric Objects (CSOs). In another project, two dual-frequency VLBI surveys of these objects were carried out to investigate the rate at which their hotspots advance.

MERLIN has been used to study the details of jets in a wide variety of extragalactic radio sources, from Seyfert galaxies and compact steep spectrum sources to QSOs and the most distant radio galaxies. Comparison of MERLIN and HST images at comparable resolution have been particularly useful. Detailed polarization observations using the VLA confirm that the asymmetric depolarization seen in radio galaxies and QSOs occurs in the surrounding medium, and further observations with MERLIN and the VLA have been used to probe the density distribution of this gas on scales of $1-100 \mathrm{kpc}$.

High-resolution ( 0.5 arcsec) BIMA imaging of the mm-wave continuum from $\mathrm{M} 87$ show detailed structure in the 20-arcsec jet as well as 1-2 arcsec clumps embedded in the extended radio lobes each side of the nucleus.

VLBI observations involving Australian, US and South African antennas have revealed a sub-parsec radio counterjet in the nucleus of NGC 5128 (Cen A). The morphology at several frequencies suggests that a disk or torus of ionized gas obscures the central half parsec of the radio source, similar to the situation believed to exist in NGC 1275.

VLBI observations of the nucleus of NGC 6251 at $1.6 \mathrm{GHz}$ show that different regions of the radio jet evolve with very different implied proper motions. This has provided additional evidence that some, if not most, component proper motions in parsec-scale jets are the results of pattern speeds rather than the bulk motions of jet material.

Third-epoch VLBI observations at $1.67 \mathrm{GHz}$ of a sample of low-frequency variable sources have shown that some have superluminal motions much like those usually observed at higher frequencies, providing some new candidates with such motions.

A model has been developed in which mildly-relativistic jets propagate through the ISM in active galaxies and collide with dense gaseous obstacles (SNRs, winds from giant stars or line-emitting clouds for example). In another model, radio jet formation in accreting objects is suppressed by processes which occur when the accretion rate approaches the Eddington limit.

Spectral Relationships. For a sample of Fanaroff-Riley type II radio galaxies it has been found that powerful radio galaxies with a steep spectrum near $1 \mathrm{GHz}$ show relatively small spectral curvature compared with those having a more normal spectrum; the curvature is greater for objects with larger core-to-hotspot separation ratios.

A Unified Theory? (See also section on Jets). The question of the existence of a unified radio galaxy/ quasar scheme has provided considerable debate. It has been suggested that although the symmetry parameters and degree of core prominence for compact steep-spectrum sources are broadly consistent with the predictions of orientation-based unified schemes, strong evidence of intrinsic asymmetries exists. To reconcile an observed excess of compact radio cores in Seyfert 2 relative to Seyfert 1 galaxies with 
the unified scheme, a model involving optical depth effects in the narrow-line region has been proposed. High-luminosity objects have been examined in terms of distributions of luminosity, projected linear size, and fraction of emission from the core (used as a statistical measure of the orientation of the source axis to the line-of-sight) and the results are found to be consistent with the scheme. The observed distribution of asymmetry angles for a sample of Fanaroff-Riley II sources was found to be consistent with the scheme, with the source components being ejected uniformly within an angle of $6^{\circ}-7^{\circ}$ about the source axis. It has been suggested that orientation may not be the main difference between radio galaxies and QSOs. By allowing for a temporal evolution of source in both size and luminosity, the claimed major discrepency with the unified scheme, namely the difference between the radio luminosity-size relations for QSOs and radio galaxies, can be explained. It has been pointed out that at metre wavelengths the fraction of QSOs in a sample appears to fall rapidly with a decrease in flux-density level of the sample, and this cannot be explained in terms of a simple orientation-based unified scheme with a fixed average value of the half-opening angle of an obscuring torus.

\subsubsection{High-z Galaxies}

The chemistry of objects at high and intermediate redshift is being studied through molecular-line absorption, often in the direction of gravitational lenses. This field has been pioneered using the SEST. Molecular absorption can be observed at any distance as long as the background source has a sufficiently strong continuum. Molecular species other than $\mathrm{CO}$ have been detected $-\mathrm{HCO}^{+}, \mathrm{HCN}, \mathrm{HNC}$, $\mathrm{CS}$, and $\mathrm{CN}$, together with isotopic variants of $\mathrm{CO}$ and $\mathrm{HCO}^{+}$. The detection of 38 different molecular transitions in four absorption systems with $\mathrm{z}=0.25-0.89$ demonstrates the potential of using molecular absorption lines to study molecular gas at large distances. The observed absorption appears to be associated with either an intervening galaxy (as for B0218+357 and PKS 1830-211) or the host continuum source (PKS $1413+135$, PKS 1830-211 and B31504+377).

$\mathrm{CO}$ emission has been observed in other high-z objects. CO J=3-2 observations of IRAS F10214+4724 at $\mathrm{z}=2.28$ with the IRAM interferometer, combined with optical and IR data showing that the galaxy is probably gravitationally lensed, suggests that the $\mathrm{CO}$ is in the extended IR arc rather than the associated compact source. In CO luminosity, molecular gas content, CO linewidth and corrected far IR luminosity, the object is a typical warm IR ultraluminous galaxy.

$\mathrm{CO}$ emission was detected with the NRAO $12-\mathrm{m}$ telescope in the radio galaxy $4 \mathrm{C} 28.55$ ( $\mathrm{z}=2.95)$. A large $\mathrm{CO}$ luminosity but narrow line profile suggests that the object is a largely gaseous galaxy in an early stage of collapse.

In other studies CO J=1-0 emission has been observed with the NRO 45-m telescope and NMA - in IRAS F10214+4724, 53W002 $(\mathrm{z}=2.39)$, and Q1235+0857 $(\mathrm{z}=2.88) . \mathrm{J}=5-4$ emission at $\mathrm{z}=4.69$ has been detected with the NMA in QSO BR 1202-0725. The luminosity for the transition is about a thousand times greater than that of our Galaxy, and the possibility that the $\mathrm{CO}$ emission is amplified by gravitational lensing cannot be excluded.

Millimetre-wave bolometers have been used in the detection of dust continuum emission in some QSOs with $\mathrm{z}$ up to 4.7

\subsubsection{Gravitational Lenses}

The study of gravitational lenses offers opportunities to constrain distributions of dark matter in the universe and determine the $\mathrm{H}_{0}$ by examining time delays between variations of individual lensed images. Radio astronomers continue to have a major influence on this growing field. In addition to discovering the majority of individual galaxy lenses, they are also able to image many of the extended lensed images in exquisite detail, so that the structure of the individual lens gravitational potential, as well as that of the intrinsic, highly magnified source brightness, can be inferred. Notable among these sources are the Einstein ring radio sources. More recent snapshot surveys of thousands of flat spectrum compact sources are uncovering more examples of multiple imaging by gravitational deflection of radio waves at about the expected rate. It is hoped that a subset of these lensed sources will be useful for making definitive physical measurements of the $\mathrm{H}_{0}$ by using the measured time lags in the variations of the individual sub images.

Building on the success of the Jodrell Bank/VLA Astrometic Survey (JVAS) aimed at locating gravitaional lens systems, a much larger survey, the Cosmic Lens All-Sky Survey (CLASS), has begun, involving Caltech, Jodrell Bank, Leiden Observatory, NFRA and JIVE. The first phase involved VLA imaging of 
3258 radio sources at $3.6 \mathrm{~cm}$. So far nine new lenses have been discovered; monitoring to determine $\mathrm{H}_{0}$ in one of them, $0218+357$ is well advanced. Over half the new lenses are four-image systems, which may have implications for the nature of the lensing galaxies involved.

It is believed that, in some cases where molecular absorption has been detected in front of QSOs, the absorption is originating from the gravitational lens. An example of this may be the SEST detection of molecular-line absorption at $\mathrm{z}=0.2$ towards a distant QSO.

The Einstein Ring PKS 1830-211 continues to be of widespread interest. VLBI observations have shown that the two compact radio components are broadened by interstellar scattering within our Galaxy; the angular size of the more compact component follows a wavelength-squared law between at least 1.6 and $22 \mathrm{GHz}$. This can explain why the brightness temperatures observed at low frequencies are unusually low. SEST observations at wavelengths near 3 and $2 \mathrm{~mm}$ have revealed a rich molecular-line absorption system at $\mathrm{z}=0.9$. BIMA images with 0.5 -arcsec resolution show that the absorption for $3-\mathrm{mm}$ transitions of $\mathrm{HCO}^{+}$and $\mathrm{HCN}$ occurs across part of the southwest continuum component but not towards the northeast component. However, line absorption at $1.19 \mathrm{GHz}$ has been detected towards the object using the Parkes telescope (and confirmed with the ATCA). If this line is interpreted as HI absorption, then the corresponding value of $z$ is only 0.19 . Subsequent ATCA observations have indicated that only the north-east continuum component is absorbed. Interpretation of all the results appears to require two galaxies along the line of sight to the background source, one 4.5 times more distant than the other.

\subsubsection{Extragalactic Source Surveys}

Many surveys of extragalactic objects have been carried out, or are in progress. The following listing includes some of the larger surveys.

A revised machine-readable source list for the Rees $38-\mathrm{MHz}$ (or $8 \mathrm{C}$ ) survey, has been produced with improved source positions and no redundancy.

The Cambridge Low-Frequency Synthesis Telescope has been used at $151 \mathrm{MHz}$ to survey with a resolution of 70 arcsec $\times 77$ arcsec a region (which includes the North Ecliptic Cap) of 418 square degrees centred at RA $17^{h}$, dec $65^{\circ} ; 157$ sources were identified with optical objects within 15 arcsec of the radio positions.

The Texas Survey of discrete radio sources at declinations between $-35^{\circ} .5$ and $71^{\circ} .5$ has been carried out at $365 \mathrm{MHz}$ with a 5 -element interferometer.

A deep 0.4-GHz survey of a $4^{\circ}$-diameter region in Hercules with the Cambridge One-Mile Telescope has yielded 232 objects brighter than $9.5 \mathrm{mJy}$, increasing to 3220 the number catalogued at this frequency in published $5 \mathrm{C}$ surveys.

A survey of two fields in the region of RA $10^{h} 30$ and dec. $+46^{\circ}$ has been made at 408 and $1420 \mathrm{MHz}$ with the DRAO Synthesis.

A survey of the southern sky south of declination $-30^{\circ}$ is planned with the MOST at a frequency of $843 \mathrm{MHz}$. The observations should reliably detect sources with flux densities down to $4-5 \mathrm{mJy}$, the level at which the population of starburst galaxies should begin to overtake the population of AGNs.

A sample of 263 Parkes half-Jansky flat-spectrum sources were found to show no evidence of clustering.

The identification of 878 flat-spectrum sources from the Parkes Catalogue has resulted in the discovery of the highest-redshift radio-selected quasar known - PKS 1251-407 with a redshift of 4.46, and confirmation of the redshift cut-off.

The first and second Caltech-Jodrell Bank VLBI snapshot surveys have imaged $\sim 300$ powerful compact radio sources with $\sim 1$ millarcsec resolution. The overwhelming majority of them are asymmetric core-jets of one form or another most of which exhibit apparent superluminal motion. However $5-10 \%$ of powerful flat-spectrum sources are 100-pc-scale CSOs; these appear to form a continuum with the $1 \mathrm{kpc}$ scale double-lobed Compact Steep Spectrum sources which make up 15-20\% of lower frequency samples. It is likely that these sub-galactic size symmetric sources are the precursors to the large-scale classical double sources. There is a surprising peak around 90 degrees in the histogram of misalignments between the dominant source axes on parsec and kiloparsec scales; this seems to be associated with sources exhibiting a high degree of relativistic beaming. VLBI snapshot surveys have great cosmological potential via measurements of both proper motion and angular size vs redshift as well as searches for gravitational milli-lensing.

Observations have been made at $21,11,6$ and $2.8 \mathrm{~cm}$ with the $100-\mathrm{m}$ Effelsberg telescope of 234 radio sources with counterparts in the ROSAT all-sky survey. No global correlation is found between the 
integrated radio flux densities and the X-ray flux densities. An improved list of flux density calibrators has been produced on the basis of observations with the telescope.

Several surveys have used the NRAO VLA. The 20-cm Sky Survey (NVSS) began in 1993 and will cover all sky between declinations -40 degrees to +90 degrees when completed by the end of 1996 . Limiting flux density is $2.5 \mathrm{mJy}$, with a beam size of 45 arcsec. Another VLA survey, Faint Images of the Radio Sky at Twenty Centimeters (FIRST) is concentrating on the north galactic cap, with emphasis on accurate radio positions for optical identifications and radio structure. Limiting flux density is $1 \mathrm{mJy}$, and the beam size is 5.4 arcsec. In a third $20-\mathrm{cm}$ survey to support the deepest portion of the ROSAT soft X-ray survey, a $29.3 \mathrm{deg}^{2}$ region around the north ecliptic pole has been mapped; 2435 sources were detected with flux densities in the range $0.3-1000 \mathrm{mJy}$.

Maps based on observations with the Green Bank 300-ft telescope have been used to compile the GB6 catalogue of 75,162 discrete sources with angular sizes of 10.5 arcmin and less, and lower flux-density cutoff at $18 \mathrm{mJy}$.

Further results of the Parkes-MIT-NRAO (PMN) southern sky survey at $4.85 \mathrm{GHz}$ have been published. These include maps for declinations between $-29^{\circ}$ and $-9^{\circ}$ and source catalogues for the equatorial and zenith zone regions.

A survey with the RATAN-600 radio telescope has been carried out at $3.9 \mathrm{GHz}$ for declinations $-1^{\circ}$ to $0^{\circ}$.

The Japanese domestic VLBI network has been used for a mm-wave surveys of compact flat- and steep-spectrum sources, to measure the size and spectral index of the core components.

Millimetre flux densities have been measured for extragalactic sources with the IRAM 30-m telescope, and the SEST. Based on JCMT observations, a set of secondary calibrators has been set up for sub-mm observations in bands centred at $2,1.3,1.1,0.8,0.45$ and $0.35 \mathrm{~mm}$. These sources have been calibrated relative to Mars and Uranus,

Optical identifications, magnitudes, redshifts and polarizations data have been compiled for radio sources in the $1 \mathrm{Jy}, \mathrm{S} 4$ and $\mathrm{S} 5$ catalogues.

A three-year monitoring program at $327-\mathrm{MHz}$ with the Ooty Synthesis Radio Telescope has yielded 19 variable and 6 possibly variable sources.

\subsubsection{Cosmic Microwave Background.}

Interest in the Cosmic Microwave Background and its anisotropies is continuing. This includes theoretical approaches such as investigation of the imprint of nonlinear matter condensations on the CMB cold dark matter model universes, and the impact of large-angle CMB anisotropies in an open universe. Further observational results have been obtained. The results of measurements of an area of sky at frequencies of 15.5 and $16.5 \mathrm{GHz}$ with the Cambridge Anisotropy Telescope (CAT) are consistent with the predictions of a standard COBE-normalized, cold dark-matter model. Other observations at 13.5 $\mathrm{GHz}$ show evidence for residual structure on a scale of about half a degree. The Jodrell Bank CMB experiments on Tenerife have located the first individual hot and cold features with a series of beamswitching observations exploring scales of 5 to 15 degree at 10,15, and $33 \mathrm{GHz}$. These individual features can be identified in the independent COBE Differential Microwave Radiometer (DMR) data at 53 and $90 \mathrm{GHz}$. In the Tenerife results, the rms amplitude of the structure is 54 micro K. Measurement of the 1.4-GHz absolute temperature of the CMB with a ground-based radiometer has yielded a value of $2.65 \mathrm{~K}$. $90-\mathrm{GHz}$ observations of a low-background region of sky at high galactic latitude has revealed significant anisotropy on degree angular scales.

Efforts are being made to correct CMB results for the effects of external phenomena. Four-year COBE DMR maps have been used to model galactic microwave emission at high latitudes and correct for this emission. A cross-correlation of COBE maps with long-wavelength radio surveys suggests that free-free emission should not significantly contaminate measurements of the cosmic microwave anisotropy at degree angular scales for frequencies above $20 \mathrm{GHz}$. A sensitive wide-field $\mathrm{H} \alpha$ image of the north celestial polar region has indicated an upper frequency limit of $27 \mathrm{GHz}$ for variations in free-free brightness temperature. The effect of structures such as the Great Attractor has been modeled.

Sunyaev-Zel'dovich Effect. Several telescopes have been used in searches for the Sunyaev-Zel-dovich effect towards clusters of galaxies. CSO observations provided the first significant detection at mm wavelengths $(2.2 \mathrm{~mm})$, towards the cluster A2163. SEST observations towards M0654015 have resulted in a 
detection at $1.2 \mathrm{~mm}$ (in emission) and at $2 \mathrm{~mm}$ (in absorption), the first time SZE has been found on both sides of the CMB peak. Other observations towards X-ray ROSAT clusters have provided marginal detections for A2744 and S1077. The OVRO 5.5-m telescope has yielded a CMB decrement at 32-GHz towards the Coma cluster (after corrections associated with the presence of discrete radio sources and an $\mathrm{X}$-ray atmosphere of the cluster), the first detection of the SZE towards a nearby cluster. The results were consistent with $\mathrm{H}_{0}=71.30-\mathrm{GHz}$ images of the SZE have been obtained with the OVRO array towards the clusters CL $0016+16$ and A773. A resolved decrement has been detected towards A1413 with the Ryle telescope.

\section{Radio Frequency Interference to Radio Astronomy}

\subsection{INTER-UNION COMMISSION ON THE ALLOCATION OF FREQUENCIES TO RADIO ASTRONOMY AND SPACE SCIENCE}

The Inter-Union Commission for the Allocation of Frequencies for Radio Astronomy and Space Science (IUCAF) is a group representing the IAU, the International Union of Radio Science (URSI), and the Committee on Space Research (COSPAR) in matters related to scientific usage of the radio spectrum. It has the status of a recognized International Organization at meetings organized by the International Telecommunication Union (ITU), and as such is particularly effective in presenting the coordinated views of the radio astronomy community on such occasions. IUCAF is particularly involved in the protection of frequency bands used by radio astronomers from the effects of interfering signals from other users of the radio spectrum. Recently, it has played a major role in efforts to protect radio astronomy observations at $1.6 \mathrm{GHz}$ from the harmful interference by transmitters on satellites (see later section).

\subsection{COMMITTEE ON RADIO ASTRONOMY FREQUENCIES}

In 1988, on behalf of the European radio astronomy community the European Science Foundation formed the Committee on Radio Astronomy Frequencies (CRAF), with the task of coordinating efforts to keep the frequency bands used by radio astronomers free from interference. Member organizations associated with CRAF are the European Space Agency, the European Incoherent Scatter Scientific Association, and the Institut de Radio Astronomie Millimetrique. CRAF operates out of an office at the Netherlands Foundation for Research in Astronomy. Meetings are held at six-monthly intervals in different countries. Issues discussed at the meetings include the impact on radio astronomy of the World Radiocommunication Conferences (WRCs) which are now held every two years, and preparations for future Conferences (WRC97 for instance). Attention is being paid to new developments and methods in spectrum management, changing regulations and new standards; pricing in spectrum usage is now a reality. Other items are the increasing threat of interference from low-Earth-orbit satellites using frequency bands near bands internationally allocated to radio astronomy, and the protection of $\mathrm{mm}$-wavelength astronomy. During the last three years, CRAF has participated in the organization of the Wroclaw EMC Symposium 1994, organized sessions at other scientific meetings, communicated with NATO regarding interference problems due to radar in Italy and Spain, and communicated with various satellite companies about interference problems and interference threat by planned broadcast and cloud radar.

\subsection{HANDBOOKS ON RADIO ASTRONOMY}

Two booklets focussing on radio astronomy as a radiocommunication service sharing the radio spectrum with other services were published in 1995. The CRAF Handbook for Radio Astronomy was published by the European Science Foundation, while the ITU Handbook of Radio Astronomy was published by the ITU.

\subsection{GLONASS AND RADIO INTERFERENCE AT $1612 \mathrm{MHZ}$}

The threat to radio astronomy from transmitters on satellites has been amply demonstrated by the case of the Russian global navigation satellite system GLONASS, which for many years has caused world-wide radio interference to observations of the important $1612-\mathrm{MHz}$ line of $\mathrm{OH}$, the characteristic radio emission from OH-IR sources. By a combination of negotiation and legislation, the problem is now being resolved. A key milestone was the upgrade in 1992 of the radio astronomy allocation in the band 1610.6-1613.8 
$\mathrm{MHz}$ from secondary to primary status, plus addition of footnote protection for radio astronomy, in the international Radio Regulations. Negotiations between IUCAF and the Russian administration led to the GLONASS-Radio Astronomy Joint Experiment in November 1992, in which satellite transmissions were varied and their impact on radio astronomy observations were monitored at several observatories around the world. Following a technical evaluation of the results, an agreement concerning frequency usage was signed between the GLONASS Administration and IUCAF on 1993 November 4.

This agreement set out a step-by-step plan for reducing interference from the GLONASS-M satellite system to radio astronomy measurements in the bands $1610.6-1613.8 \mathrm{MHz}$ and $1660-1670 \mathrm{MHz}$. By 1998 the main (narrow-band) GLONASS emission will be excluded from the former band. This is already happening, and the interference levels are reduced, even though the full complement of 24 satellites is now deployed. From 1999 the main (broadband) will also be excluded, which should produce a significant second reduction in interference. The agreement also outlines further measures to reduce interference levels, including installation of filters on the satellite transmitters and a further reconfiguration of the satellite frequencies. The steps of the agreement have been formalized in a series of coordination negotiations between the Russian administration and administrations of other countries. Indications are that the steps will be met by the year 2005 .

Assuming that all goes well, the GLONASS problem will have taken over twenty years to resolve. One of the spinoffs is that the footnote added to the Radio regulations in 1992 to protect the $1612-\mathrm{MHz}$ $\mathrm{OH}$ line is now helping to keep new sources of interference at bay. For instance, the IRIDIUM satellite system is poised to start 'uplink' transmissions in bands near and including the radio astronomy band, and 'downlink' transmissions at frequencies not far above the radio astronomy band. However, because of the protection built into the Radio Regulations, the system must operate so as to avoid interference to radio astronomy observations in the band 1610.6-1613.8 MHz. Negotiations are underway between the radio astronomy community (including IUCAF) and Motorola to establish how this goal can be met.

\subsection{SEARCHES AND MONITORING AT $1612 \mathrm{MHZ}$}

The HartRAO telescope has been used since 1985 to monitor 1612-MHz maser emission from OH/IR stars. In September 1993 the central frequencies of the GLONASS satellites were moved away from the $1612-\mathrm{MHz} \mathrm{OH}$ band. However, observations in the band were still being degraded by the 5.11-MHz CAM signal of GLONASS satellites transmitting anywhere between $1607 \mathrm{MHz}$ and $1617 \mathrm{MHz}$. In November 1994 three new satellites, transmitting at $1608.7500 \mathrm{MHz}, 1609.3125 \mathrm{MHz}$ and $1613.8125 \mathrm{MHz}$, were launched, adding extra interference to the radio astronomy band. The satellite transmitting at 1613.8125 $\mathrm{MHz}$ was launched in anti-phase with the another transmitting at the same frequency, making the available ' $1612-\mathrm{MHz}$ observing windows' of very short duration. Because of the high interference, the HartRAO $\mathrm{OH} / \mathrm{IR}$ monitoring program was terminated in mid-1996.

\section{Acknowledgements}

This report could not have been accomplished without the assistance of Commission 40 members. Contributions or assistance from the following members are gratefully acknowledged: $\mathrm{K}$ Anantharamaiah, W. Baan, D. Backer, R. Beck, E. Berkhuijsen, R. Blandford, R. Booth, J. Cohen, F. Combes, R. Dagkesamanskii, R. Davis, H. Dickel, G. Dubner, R. Duncan, E. Feigelson, L. Feretti, M. Garrett, G. Giovannini, A. Green, M. Grewing, D. Harris, J. Heidmann, C. Heiles, M. Ishiguro, D. Jauncey, D. Jones, H. Kahlmann, B. Koribalski, T. Kuiper, T. Landecker, K. Lo, F. Lockman, R. Manchester, J. Moran, D. Morris, L. Padrielli, A. Sargent, R. Sault, M. Reid, L. Staveley Smith, G. Swarup, Y. Terzian, P. Vanden Bout, W. Van Driel, M. West, R. Wielebinski, M. Wieringa and S. Ye. 